OPEN ACCESS

Edited by:

Bin Chen,

New Jersey Institute of Technology,

United States

Reviewed by:

Hamish Andrew Sinclair Reid,

University College London,

United Kingdom

Nat Gopalswamy,

Goddard Space Flight Center,

National Aeronautics and Space

Administration, United States

*Correspondence:

Karl-Ludwig Klein

ludwig.klein@obspm.fr

Specialty section: This article was submitted to

Stellar and Solar Physics,

a section of the journal

Frontiers in Astronomy and

Space Sciences

Received: 06 July 2020

Accepted: 23 November 2020

Published: 12 February 2021

Citation:

Klein K-L (2021) Radio Astronomical

Tools for the Study of Solar Energetic

Particles I. Correlations and

Diagnostics of Impulsive Acceleration

and Particle Propagation.

Front. Astron. Space Sci. 7:580436.

doi: $10.3389 /$ fspas.2020.580436

\section{Radio Astronomical Tools for the Study of Solar Energetic Particles I. Correlations and Diagnostics of Impulsive Acceleration and Particle Propagation}

\author{
Karl-Ludwig Klein *
}

Observatoire de Paris, LESIA and Station de Radioastronomie de Nançay, Univ. PSL, CNRS, Sorbonne Univ., Univ. de Paris, Univ. d'Orléans, Meudon, France

Solar energetic particles (SEPS) are sporadically ejected from the Sun during flares and coronal mass ejections. They are of major astrophysical interest, because the proximity of the Sun allows for detailed multi-messenger studies. They affect space weather due to interactions with electronics, with the Earth's atmosphere, and with humans if they leave the protective shield of the magnetosphere of the Earth. Since early studies in the 1950s, starting with particle detectors on the ground, SEP events have been related to radio bursts. Two subjects are addressed in this chapter: attempts to establish quantitative correlations between SEPs and microwave bursts produced by gyro synchrotron radiation of mildly relativistic electrons, and the information derived from type III radio bursts on impulsive processes of particle acceleration and the coronal and interplanetary propagation. Type III radio bursts produced by electron beams on open magnetic field lines have a wide range of applications, including the identification of acceleration regions, the identification of confined particle acceleration with coronal signatures, but no SEPs, and the paths that the electrons, and energetic charged particles in general, take to travel from the low corona to the Heliosphere in case they escape. Simple scenarios of coronal particle acceleration are confirmed in relatively simple and short events. But the comparison with particle transport models shows that longer and delayed acceleration episodes exist especially in large SEP events. They will be discussed in a companion chapter.

Keywords: acceleration of particles, sun: particle emission, sun: radio emission, sun: flares, sun: coronal mass ejections

\section{INTRODUCTION}

Solar energetic particles (SEPs) is the term used for energetic particles observed in situ in the Heliosphere, which are accelerated during solar flares and coronal mass ejections (CMEs). They are a major element of space weather disturbances (Knipp, 2011; Schwadron et al., 2017; Malandraki and Crosby, 2018), because they interact with space-borne electronics, possibly with airborne electronics, and add to galactic cosmic rays as a source of radiation in the Earth's atmosphere. They are a serious threat for astronauts outside the magnetosphere. From the astrophysicist's viewpoint SEPs are an 
illustration of charged particle acceleration in the Universe that can be studied with much detail. Observations in X-rays, EUV and white light trace the plasma dynamics in the corona where particle acceleration takes place. Gamma-ray, hard X-ray and radio observations probe energetic electrons and protons in the solar atmosphere and can be combined with in situ measurements to determine the acceleration regions and the processes that govern the propagation of SEPs in the corona and the Heliosphere. All this can be done with time resolutions relevant to the evolution of acceleration and propagation in individual events.

Radio bursts and energetic particles from the Sun were discovered in 1942. Since both are related to non-thermal particles during sporadic events of solar activity, the link between the two phenomena was rapidly established, and radio bursts became a tool to explore the origin of SEPs since the 1950s. At that time the most obvious transient process in the corona were flares and filament eruptions. The advent of spaceborne coronagraphs drew attention to the importance of CMEs as drivers of shock waves that can accelerate SEPs in the high corona and the interplanetary medium (e.g., Reames, 1999; Gopalswamy, 2009; Desai and Giacalone, 2016; Schwadron et al., 2017). Different aspects of SEP events were reviewed in Klecker et al. (2006), Reames (2015), Simnett (2017) and Klein and Dalla (2017).

Radio emission provides key information related to the acceleration and propagation of energetic particles. The degree of correlation between SEPs in space and signatures of nonthermal particles in the solar atmosphere can be used to constrain physical relationships between the particle populations. One such signature are microwave bursts (frequency above $1 \mathrm{GHz}$, wavelengths shorter than $30 \mathrm{~cm}$ ), produced by mildly relativistic electrons through gyrosnchrotron emission in the low corona. Radio bursts at longer wavelengths are understood to reveal features such as the presence of field lines that connect the corona with the Heliosphere (type III bursts), shock waves (type II bursts), and confined electron populations (type IV bursts), which may all be relevant to understand the connection between eruptive processes in the solar corona and particle populations in space. The reader is referred to other chapters in this volume for presentations of radio bursts (Carley et al., 2020; Reid, 2020; Vourlidas et al., 2020).

This chapter starts with a brief account of the history of joint radio and in situ studies of SEPs in Section 2, and discusses correlations between quantitative measures of the importance of SEP events and microwave bursts. The use of type III bursts to probe particle acceleration and particle propagation through the corona and interplanetary space is addressed in Sections $\mathbf{3}$ and $\mathbf{4}$, respectively. Type III radio emission is produced at the electron plasma frequency or its harmonic by electron beams. The electrons are accelerated to energies in the $\mathrm{keV}$ to tens of $\mathrm{keV}$ range in impulsive processes and injected onto open magnetic field lines that connect the parent active region with the Heliosphere. Tracing the emission through the radio spectrum allows us to follow the electrons from the low corona, at frequencies of about a $\mathrm{GHz}$, to the vicinity of the Earth (frequency near $20 \mathrm{kHz}$ ). Time-extended particle acceleration including relativistic energies and its relationship with bursts of types II and IV will be addressed in a companion chapter (Klein, 2021).

\section{EMPIRICAL RELATIONSHIPS BETWEEN SOLAR ENERGETIC PARTICLES AND RADIO EMISSION IN THE CORONA}

\subsection{SEPs and Radio Bursts - A Brief Historical Overview}

The first SEP observations were either made from ground, when the SEP spectrum extended to $\mathrm{GeV}$ energies, or through the ionospheric absorption due to excess ionization by SEPs at tens of $\mathrm{MeV}$ impacting the polar ionosphere of the Earth. Balloon-borne and rocket-borne observations followed. The association between SEPs and solar radio emission was made rapidly when synchrotron emission of relativistic electrons was believed to be the radiation process of type IV bursts, which are broadband emissions at meter wavelengths, sometimes extending up to $\mathrm{cm}$-wavelengths, with durations that may reach several tens of minutes or even hours (see the second panel from top in Figure 4B). A physical relationship was supported by the coexistence of two prominent manifestations of solar activity, the estimate of comparable numbers of relativistic electrons in the type IV source and of relativistic protons at Earth (Boischot and Denisse, 1957), and by similar time profiles of the type IV emission at frequencies below $100 \mathrm{MHz}$ and the SEP intensity at energies near $170 \mathrm{MeV}$ (Figure 2 in Boischot and Warwick, 1959). A delay of the type IV onset with respect to the early flare signatures in $\mathrm{Ha}$ was considered as an indication of a rising source that connected the chromosphere, where the flare was then supposed to have its origin, with the high corona and the interplanetary space. The first years of research on this topic are reviewed in Section 3.21 of Wild et al. (1963) and in more detail in chapter 14 of Kundu (1965) (see also Section 2.1.3 of Pick and Vilmer, 2008).

An early interpretation of the association between type IV bursts and SEP events (Boischot and Denisse, 1957; Hakura and Goh, 1959) was that the moving type IV burst was the signature of a plasma cloud released from the flaring active region. Relativistic electrons confined within the cloud were to produce the type IV burst by synchrotron emission, while the turbulence within the cloud was to accelerate protons to high energies, which escaped from the cloud when the energy was high enough, and therefore arrived at Earth before the cloud itself. The cloud was supposed to trigger a geomagnetic storm and in some cases a Forbush decrease of galactic cosmic rays. The picture is very close to the present idea that CMEs are a key ingredient in SEP events. The interpretation incorporates a time delay between the start of the flare and the release of accelerated particles into the Heliosphere. Wild et al. (1963) proposed a scenario (their Section 3.23) in two phases, where the first phase, i.e., the impulsive flare phase, was a general counterpart of solar flares, while the second phase, where relativistic electrons and protons were accelerated, occurred only in strong flares, but was directly 
triggered by the first phase. They ascribed the second-phase acceleration of relativistic electrons and protons to a MHD shock wave shown by its narrow-band radio emission with slow drift toward lower frequencies (type II burst; two top panels in Figure 4B). This picture became very influential later in the discussion on the origin of large SEP events (Reames, 1999). The empirical association between SEP events and type II and type IV bursts is still a tool for the forecasting of SEP events (e.g., Balch, 2008).

\subsection{Quantitative Correlation}

Correlation studies with radio bursts especially at meter wavelengths remained for some time the basic tool of research on the origin of SEP events. Kundu and Haddock (1960) found that while most SEP events at tens of MeV were accompanied by type IV bursts, a few were only accompanied by type II bursts. However, all were found to have a strong microwave counterpart. Microwave emission in strong bursts is gyro-synchrotron emission from mildly relativistic electrons (see Nindos, 2020) and is therefore a priori a better indicator of particle acceleration than the widely used thermal soft X-rays. The flux density of microwave emission had actually been recognized as a criterion that distinguishes between SEP-events and non-SEP events in the early 1960s (Avignon and Pick-Gutmann, 1959; Kundu and Haddock, 1960; Kundu, 1965). The SEP-associated microwave bursts were found to have long duration (Kundu and Haddock, 1960; Sakurai and Maeda, 1961; Kahler, 1982a), which means that the microwave emission is in general the high-frequency part of a type IV burst. The necessity of both microwave and meter-wave emission, which signals the escape of particles to the high corona, was emphasized by Castelli et al. (1967), Akinyan et al. (1971), and Castelli and Barron (1977).

One may wonder why there should be a relationship between protons and ions in space, and microwaves emitted by nonthermal electrons in the corona. From an empirical viewpoint protons and ions at tens of $\mathrm{MeV}$ are strongly correlated with nearrelativistic and mildly relativistic electrons in space (Daibog et al., 1989; Posner, 2007; Trottet et al., 2015). The early belief that the acceleration processes of protons and electrons in solar flares were intimately connected was, however, challenged by the RHESSI observation that hard X-rays from near-relativistic electrons and gamma-ray lines from deka- $\mathrm{MeV}$ nucleons have slightly, but significantly, different sources (Hurford et al., 2006; Vilmer et al., 2011). But Fomichev and Chertok (1985) and Chertok (1990) demonstrated a correlation between the peak flux of microwave bursts (at $15.4 \mathrm{GHz}$ ) and nuclear gamma-ray line peak fluxes in solar flares. Similarly, Shih et al. (2009) found a correlation between hard X-rays from electrons above $300 \mathrm{keV}$ and gamma-ray line intensities. This means that microwave flux densities may be considered as a measure of gamma-ray line intensities, too, and reinforces the interest to use the more abundant microwave measurements in correlation studies with SEP intensities.

Another caveat of using gyrosynchrotron emission as a quantitative measure of electron acceleration is the potential action of self-absorption. It is generally argued that the higher the frequency, the more likely the source is optically thin, so that the flux density is directly related to the number of non-themal electrons. Croom (1971) showed that spectra of SEP-associated microwave bursts have their maximum at higher frequencies than bursts with no SEP-association. Even at the highest microwave frequency that is routinely monitored, $35 \mathrm{GHz}^{1}$, only half of the bursts can be considered to be optically thin (Correia et al., 1994). Self-absorption will hence affect a possibly existing relationship between microwaves and SEPs. Nonetheless a number of studies showed that microwave peak fluxes and fluences correlate with SEP peak intensities at tens of MeV (Kahler, 1982b; Chertok, 1990; Daibog et al., 1993; Isaeva et al., 2010; Grechnev et al., 2013; Trottet et al., 2015).

Grechnev et al. (2015) considered SEPs at higher energies than the usual tens of $\mathrm{MeV}$ analyzed before. They found that the integral parameters microwave fluence at $35 \mathrm{GHz}$ and proton fluence above $100 \mathrm{MeV}$ are more strongly correlated than the instantaneous parameters microwave peak flux and proton peak intensity. Their interpretation is that the high-energy protons tend to be accelerated by a mechanism that is closely related to the acceleration of the near-relativistic electrons producing the emission at $35 \mathrm{GHz}$-a process they term flare-acceleration. The authors consider that some proton-rich outliers may reveal predominant acceleration by the CME shock. Cliver (2016) contradicted the conclusion that the outliers could be treated as distinct phenomena, and argued in favor of a general acceleration of SEPs by the CME shock.

Chertok et al. (2009) proposed to use microwaves to predict the hardness of SEP energy spectra. They showed that SEP events with hard proton spectra between 10 and $100 \mathrm{MeV}$ tend to be accompanied by microwave bursts that are particularly strong at high frequencies. They used the two highest frequencies where the flux density is monitored continually, 8.8 and $15.4 \mathrm{GHz}$ (Radio Solar Telescope Network RSTN of the US Air Force). Proton spectra with index $<1.5$ (hard spectra) are found to be associated with microwave bursts with spectral peak above $8.8 \mathrm{GHz}$. The ratio of flux densities at the two frequencies, which is an easily observable parameter, correlates with the proton spectral hardness. The correlation has a broad scatter, but could again be confirmed by the SEP events in September 2017 (Chertok, 2018).

The existence of a correlation between two parameters is of course not a sufficient condition to infer a direct physical relationship between the two phenomena. Different eruptive manifestations in the solar corona such as soft X-ray peak flux and fluence, CME kinematics, and microwave peak flux and fluence, are all correlated with each other (Trottet et al., 2015), a phenomenon that Kahler (1982b) termed the 'big flare syndrome'. Multi-parameter statistics could in principle help to remove such internal correlations, but the events are too few to obtain detailed results (Trottet et al., 2015). The representation of SEP events by measurements in a single point is also a problem, although some studies attempt to correct for this. It is unclear whether statistical correlations

${ }^{1}$ Univesity of Berne until 2004, Nobeyama radio observatory. 
are unable to decide between an exclusive or dominant acceleration of SEPs in flare-like processes and at CMEdriven shocks, or whether they show that such a distinction simply does not exist. It is also important to consider that correlations of SEP parameters with coronal activity may vary with the energy of the SEPs: Dierckxsens et al. (2015) found a higher correlation of SEP intensity with CME speed than with soft X-ray peak flux at SEP energies below $20 \mathrm{MeV}$, and the inverse at higher energies.

\section{TYPE III BURSTS AS TRACERS OF PARTICLE ACCELERATION}

Type III bursts are a reference radio emission for SEP studies, because they are frequently observed and because they indicate open magnetic field lines. The advent of space-borne radio spectrographs provided nearly seamless observations from the low solar corona, where the bursts are emitted at decimetric wavelengths $(\sim 1 \mathrm{GHz})$, to the spacecraft, with a typical frequency of $20 \mathrm{kHz}$ (wavelength $15 \mathrm{~km}$ ). In this section the role of type III bursts as probes of particle acceleration is discussed.

\subsection{Radio Emission and SEPs During Simple Impulsive Events}

\subsubsection{An Illustration: May 1, 2000}

The flare of class M1.1 on May 1, 2000 is a showcase event, where different tools combining in situ and remote sensing techniques can be combined to get insight into the source region and propagation of energetic particles. Figures $\mathbf{1 A}-\mathbf{C}$ display the time evolution of the radio emission from centimetric (C) to hectometric (A) wavelengths. The light curves (C) show a broadband microwave burst due to gyrosynchrotron emission of electrons of hundreds of $\mathrm{keV}$ to perhaps a few $\mathrm{MeV}$. The emission comes from loop structures in the flaring active region (Nindos, 2020). A hard X-ray burst with a photon spectrum up to several hundreds of keV (Figure 1 of Kartavykh et al., 2007) provides complementary evidence on the acceleration of mildly relativistic electrons during this event. The group of type III bursts in the dynamic spectrum at meter-wavelengths (B) demonstrates that electrons escape to the overlying corona. The type III bursts seem to have different start frequencies, but this may also be a threshold effect due to their different flux densities. In the decameter-to-hectometer (henceforth abbreviated $\mathrm{DH}$ ) range these bursts merge into a few strong type III bursts at frequencies below $14 \mathrm{MHz}$ (Figure 1A). The type III bursts in this panel without counterpart above $30 \mathrm{MHz}$ are

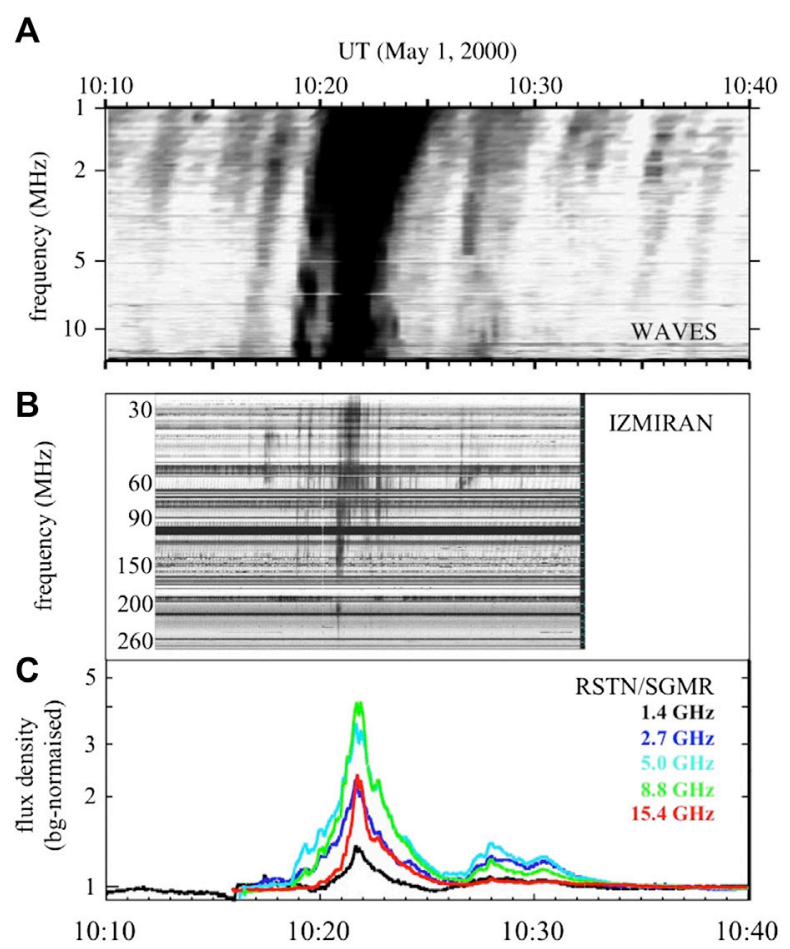

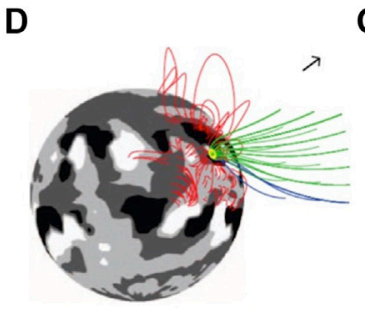

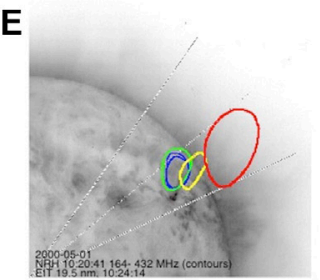

$\mathbf{F}$

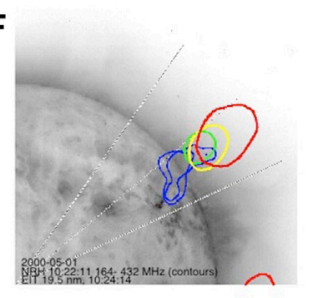

G

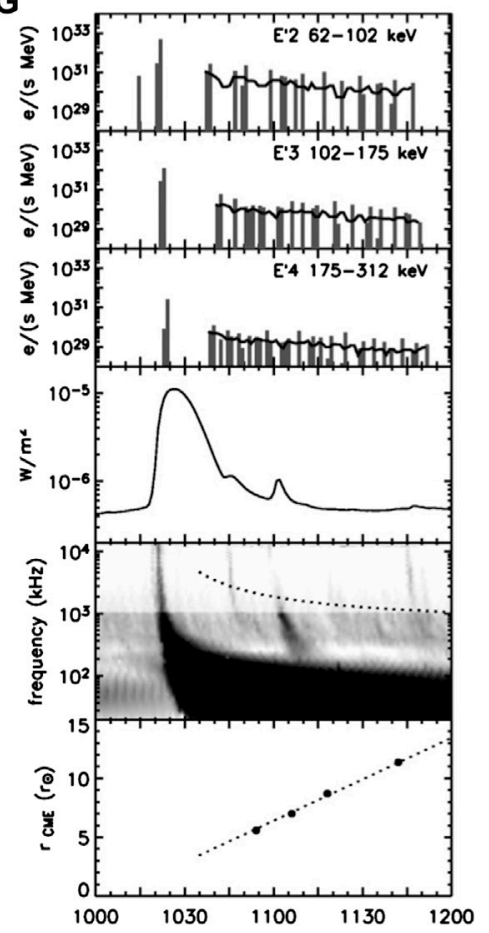

FIGURE 1 | Radio observations, magnetic field mapping, and electron release modeling during the May 1, 2000 flare. Left column: Dynamic spectra at decameter ((A); Maia and Pick, 2004, OAAS) and meter wavelengths (B); IZMIRAN, https://www.izmiran.ru/stp/lars/; figure provided by I. Chertok and R. Gorgutsa) and time histories at discrete microwave frequencies ((C); data provided by NGDC, https://www.ngdc.noaa.gov/stp/space-weather/solar-data/solar-features/solar-radio/rstn-1second/). Middle column: synoptic map of the photospheric magnetic field and field lines extrapolated using a PFSS model ((D); Wang et al., 2006b, (OAAS) and iso-intensity contours of the radio sources during two type III bursts overlaid on an EUV image at $19.5 \mathrm{~nm}$ wavelength ((F); after Klein and Posner, 2005, (OESO). The two radial lines through the disk center delimit the CME as reported by Kahler et al. (2001). Right column (G): Injection functions of electrons in three channels of ACE/EPAM, compared with the soft X-ray time profile (third from bottom), the dynamic radio spectrum in the range $14 \mathrm{MHz}-120 \mathrm{kHz}$ (frequency increasing from bottom to top on the vertical axis), and the height-time plot of the CME (bottom). From Agueda et al. (2008), OAAS. Figures reproduced with permission. 
unrelated with the flare under study. This impulsive flare is consistent with a simple scenario of the origin of SEPs: acceleration during a flare in an active region, release to the chromosphere (hard X-rays) and into low coronal loops (microwaves), escape to the Heliosphere along open magnetic field lines. A similar event is discussed in Section 22.3 of Vilmer (2011).

Figure 1D shows the magnetic field configuration around the flaring active region as inferred from a potential field model with a solar wind source surface (Wang et al., 2006b; Nitta et al., 2006). The parent active region has open field lines extending to the ecliptic plane (plotted in blue). The geometry of the radio sources during two type III bursts at meter wavelengths, observed by the Nançay Radioheliograph (NRH), is overlaid in Figure $\mathbf{1 E}$ and $\mathbf{1 F}$ on an EUV image of the corona taken by SoHO/EIT. The two type III bursts have slightly different sources, showing electrons that travel along different paths in the high corona in different bursts. The open field lines plotted in green do not connect to the ecliptic plane.

Energetic particles observed near $1 \mathrm{AU}$ by the ACE and Wind spacecraft were studied in detail in many publications, and different approaches were used to determine their solar release time. The results are summarized in Table 1. The simplest approach to infer the release time at the Sun of the first particles that are detected in situ is the ballistic back-projection of the onset time of the electron event at a given energy at the spacecraft, assuming an interplanetary travel distance. This approach is labeled BAL in the third column of the Table. If the electrons are detected in a sufficient number of energy channels, one can use the start time as a function of energy to trace their arrival time at the spacecraft, $t_{\mathrm{sc}}$, to the solar release time, $t_{\mathrm{SRT}}$, using the assumption that electrons of any energy are released at the same point and the same instant in the corona, and that they travel the same distance $L: t_{\mathrm{sc}}=t_{\mathrm{SRT}}+L / v . v$ is the speed of the electrons. This technique is called velocity dispersion analysis (VDA). Both the VDA and BAL techniques replace the complex particle transport in the time-variable interplanetary magnetic field by simple assumptions on the travel path. Both estimate only the release time of the first particles seen at the spacecraft, and tell nothing about the duration of the release. The widths of time intervals for the BAL and VDA techniques are the estimated uncertainties of the initial solar release. Numerical models describe the transport processes, often within the focused transport model, which considers one-dimensional propagation including the focusing of particles along the magnetic field direction by the conservation of the magnetic moment in a Parker-spiral type magnetic field model, and pitch angle scattering by the interplanetary magnetic field. These models are labeled TMod in the Table.
Within the intrinsic uncertainties of the methods the release times of the first electrons in Table 1 agree with the first type III bursts in Figure 1. The most sophisticated approach to determine solar release, using numerical transport models, leads to time-extended release episodes which agree reasonably with the overall timing of the group of type III bursts. One reason that the simple methods estimating the initial electron release work well here is that the pitch-angle scattering is low, as demonstrated by the long mean free paths inferred from the transport models (Kartavykh et al., 2007; Agueda et al., 2008).

Table 1 shows that the first deka-MeV protons and the first heavy ions at energies below $1 \mathrm{MeV} /$ nuc may also be released in the impulsive phase, but within broader uncertainties than the electrons. The modeling of the heavy ions actually uses data with $1 \mathrm{~h}$ integration time, so that the inferred timing is less constrained than for the electrons. Remarkably, protons of lower energy seem to be released much later. Figure 7 of Kartavykh et al. (2007) shows that a weak early release is not represented by the model, but the bulk of the protons is clearly released later, at about the time when Agueda et al. (2008) infer a late time-extended electron release (10:36-11:52 UT). This later release is accompanied by a few faint DH type III bursts and weak soft X-ray enhancements (Figure 1G), while the flare is still visible in SoHO/EIT images. Judging from the images taken by the NRH (not shown), the burst locations are related with the same active region as the stronger type III bursts in the impulsive phase, but are more widely scattered.

A jet or narrow CME was observed by the SoHO/LASCO coronagraph (Kahler et al., 2001; Wang et al., 2006b) with a speed in the plane of the sky of $1,360 \mathrm{~km} \mathrm{~s}^{-1}$. From a study of a sample of such events related with impulsive SEP events Wang et al. (2006b) find that the white-light feature propagates along a different path than the accelerated electrons, and that in particular the narrow CME on May 1, 2000 does not intercept field lines connected to the ecliptic plane. The authors therefore discard a role of the narrow CME in the acceleration of energetic particles observed near Earth. Acceleration near the CME front of the first $\mathrm{MeV}$ to deka$\mathrm{MeV}$ protons detected near $1 \mathrm{AU}$ is also inconsistent with the timing of the narrow CME and the type III bursts: Klein and Posner (2005) report that the CME front is about half a solar radius above the region where the radio images show the origin of the type III bursts. However, the fast CME provides the possibility of delayed acceleration in the corona, which offers interpretations of the late signatures of energetic electrons and protons detected in the May 1, 2000 event and many others (see Section 3.2).

TABLE 1 | Particle release times in the May 1, 2000 ent (with $500 \mathrm{~s}$ added to allow for comparison with electromagnetic observations from 1 AU).

\begin{tabular}{|c|c|c|c|c|}
\hline Species & Instrument & Method & Release time & References \\
\hline e $40 \mathrm{keV}$ & ACE/EPAM & TMod & $10: 14-10: 24$ & Kartavykh et al. (2007) \\
\hline \multirow[t]{2}{*}{ e $62-312 \mathrm{keV}$} & ACE/EPAM & TMod & $10: 20-10: 24$ & Agueda et al. (2008) \\
\hline & & & $10: 36-11: 52$ & Agueda et al. (2008) \\
\hline e $175-312 \mathrm{keV}$ & ACE/EPAM & BAL & $10: 23 \pm 1 \mathrm{~min}$ & Maia and Pick (2004) \\
\hline e $30-500 \mathrm{keV}$ & Wind/3DP & VDA & $10: 19.5 \pm 5.5 \mathrm{~min}$ & Klein et al. (2005) \\
\hline $\mathrm{Fe} 0.08-0.91 \mathrm{MeV} / \mathrm{n}$ & ACE/ULEIS & $\mathrm{BAL}$ & $10: 20 \pm 15 \min$ & Mason et al. (2004) \\
\hline $\mathrm{Fe} 0.08-0.91 \mathrm{MeV} / \mathrm{n}$ & Wind/STEP & TMod & $10: 14 \pm 15 \min$ & Kartavykh et al. (2007) \\
\hline p 0.72-1.4 MeV & Wind/3DP & TMod & $10: 50$ & Kartavykh et al. (2007) \\
\hline p 4-54 MeV & SoHO/EPHIN & VDA & $10: 24 \pm 3 \min$ & Klein and Posner (2005) \\
\hline
\end{tabular}




\subsubsection{Ambient Density and Location of the Acceleration Region}

Through the interpretation of plasma emission the starting frequency of the type III bursts determines the ambient electron density in the region where the beams start to emit (Reid et al., 2014). This is likely a lower limit of the local electron density in the acceleration region. The IZMIRAN spectrum in Figure 1B shows that the brightest type III burst starts near or above $270 \mathrm{MHz}$ in the May 1, 2000 event. The NRH sees the type III bursts at 237 and $164 \mathrm{MHz}$. Their weak polarisation suggests harmonic plasma emission, so the start frequency near $300 \mathrm{MHz}$ implies an electron density of about 3 . $10^{8} \mathrm{~cm}^{-3}$. The type III group studied by Vilmer et al. (2002) and Vilmer (2011) is very similar with a start near $300 \mathrm{MHz}$. In this event the close connection between the hard X-ray emission in the chromosphere and the decimetric radio emission in the overlying corona was underlined by coordinated changes in the imaged source structure in both spectral ranges. As pointed out, e.g., by Aschwanden (2002), Vilmer (2011), and White et al. (2011), the observations are consistent with the typical scenario of acceleration above the summits of soft X-ray loops in flares, where downward-precipitating electrons yield hard X-ray emission in the dense low atmosphere, while outward-traveling electron beams emit the type III bursts.

In some cases the radio emission comprises bursts with similar behavior as type III bursts, but which drift toward higher frequencies. The straightforward interpretation is that ordinary type III bursts are produced by upward traveling electrons, and bursts with reversed drift by downward propagating electrons, and that the acceleration region is in between. Early results of the technique are summarized in Section 3.6 of Aschwanden (2002). He inferred thermal electron densities that varied from event to event in the range $(1-10) \cdot 10^{9} \mathrm{~cm}^{-3}$. The technique was more recently applied to a sample of nine solar flares by Tan et al. (2016). From burst pairs observed in the impulsive flare phase the authors derived electron densities in the range $(6-28) \cdot 10^{9} \mathrm{~cm}^{-3}$.

Some type III bursts (e.g., Chen et al., 2013) start at higher frequencies, with an inferred thermal electron density of $7 \cdot 10^{9} \mathrm{~cm}^{-3}$ at the base of the open field lines. The authors devise a geometric model using the EUV observations of a jet and the position of the hard X-ray source to show the inclination of the open field lines along which the electron beams travel. They conclude that the acceleration region is at a height of about $15-20 \mathrm{Mm}$ above the photosphere. From a completely different approach based on the energydependent timing of individual peaks of hard X-ray emission, Aschwanden and coworkers (see Section 3.3 of Aschwanden, 2002, and references therein) inferred a typical height of $44 \mathrm{Mm}$. These values illustrate a range of heights in different flares, which Reid et al. (2014) find to be between 25 and $180 \mathrm{Mm}$. Impulsive electron events coming from much higher coronal regions are actually more frequent, since many events show power-law spectra without any turnover down to 1-2 keV (Potter et al., 1980; Lin, 1985; Kahler, 2007). Such a turnover would be created by Coulomb collisions with the ambient electrons if the escaping electrons had traveled through a dense plasma. Lin (1985) concluded that the acceleration region had to be located at least $350 \mathrm{Mm}$ above the photosphere. Evidence for the combined acceleration at low and high $\left(\sim 1 R_{\odot}\right)$ coronal altitudes was seen in a case study of bidirectional radio bursts at meter wavelengths (Klein et al., 1997), and high-coronal acceleration regions were inferred earlier from observations with the Culgoora Radioheliograph (Wild, 1968). These events of high coronal electron acceleration seem to be the low-energy manifestation of acceleration processes related to flare-like energy conversion, i.e., magnetic reconnection that occurs in fully developed flares, at the release of jets, and during the evolution of coronal streamers (see also the discussion and references in Kahler, 2007).

Since the timing of the initial release of electrons and ions seems consistent in Table 1, one can compare these ambient densities with values inferred from the charge states of $\mathrm{Fe}$ measured in impulsive SEP events. High charge states of Fe are a characteristic feature of impulsive SEP events, and were for some time attributed to high temperatures, of order $10 \mathrm{MK}$, thought to be typical for the impulsive flare phase (Reames, 1999). It was later recognized that the charge states depended on energy (Klecker et al., 2007; DiFabio et al., 2008). This can be explained by collisional stripping of the ions during their acceleration (Kocharov et al., 2000, 2001). The process can be modeled, and the measured charge states constrain the product of the ambient particle density $n$ and the residence time in the acceleration region, $\tau$. For the event on May 1, 2000 Kartavykh et al. (2007) infer two acceleration regions, which may be extreme values for a continuum in between: a cool region $\left(10^{6} \mathrm{~K}\right)$ where $n \tau=9$. $10^{10} \mathrm{~cm}^{-3} \mathrm{~s}$, where the bulk of the ions is accelerated, and a minor contribution from a second region with $T=1.58 \cdot 10^{7} \mathrm{~K}, n \tau=$ $10^{11} \mathrm{~cm}^{-3} \mathrm{~s}$. If these ions were accelerated in the same region as the electrons emitting the type III-bursts, the residence time would be $\tau=10^{11} / 3 \cdot 10^{8} \mathrm{~s} \simeq 5 \mathrm{~min}$. This duration is not inconsistent with the initial release times of the ions in Table 1. However, comparative studies of nuclear gamma-ray emission and of hard X-ray emission of electrons usually show that the two particle species evolve together on time scales much closer to $1 \mathrm{~s}$ than to $5 \mathrm{~min}$ (Forrest and Chupp, 1983; Kane et al., 1986; Vestrand et al., 1999; Kiener et al., 2006; Vilmer et al., 2011). So $\tau$ could not be the typical acceleration time, but a trapping time in dense coronal structures, before the ions escape. Since ambient densities as high as $10^{11} \mathrm{~cm}^{-3}$ are not common in impulsive flares, although they may be encountered occasionally in coronal thick target hard X-ray sources (Veronig and Brown, 2004; Fletcher et al., 2011), the trapping time would have to exceed by far $5 \mathrm{~min}$.

The frequent occurrence of impulsive ${ }^{3} \mathrm{He}$-enriched SEP events with electron events that show no bending due to Coulomb collisions led Cliver and Kahler (1991) and Wang et al. (2016) to propose acceleration much higher in the corona, where the time scales of ion acceleration would be much longer. While the detailed independent analyses of the May 1, 2000 event and some others (Pick et al., 2006) give evidence on the coordinated acceleration of electrons from ten to hundreds of $\mathrm{keV}$, ions at $(0.1-1) \mathrm{MeV} / \mathrm{s}$, and deka-MeV protons in the corona during the impulsive flare phase, a spectrum of scenarios is probably realized in different events.

\subsubsection{Evidence on Fragmented Energy Release and Particle Propagation in a Fibrous Corona}

A type III burst at decametric and longer wavelengths is not a simple entity that can be ascribed to an individual electron beam. It has been shown repeatedly that single bursts in this spectral range 
result from the merging of several bursts at higher frequencies (e.g., Lin et al., 1973; Poquérusse et al., 1996). Even relatively simply structured type III bursts at $\mathrm{DH}$ wavelengths, produced during inconspicuous activity in EUV, result from complex activity in the low corona (Alissandrakis et al., 2015). The type III bursts in Figure 1 also involved different coronal field lines.

Chen et al. (2013) used dynamical spectroscopic imaging with the VLA for a very detailed analysis of a group of type III bursts at decimeter wavelengths $(1.0-1.5 \mathrm{GHz})$, i.e., lower in the corona than during the May 1, 2000 flare. The bursts accompanied a hard $\mathrm{X}$-ray burst at photon energies $12-25 \mathrm{keV}$ and a jet in EUV, both typical signatures of impulsive energy release in the corona. The source centroids of the bursts are plotted in panel (A) of Figure 2. The frequencies are coded by colors from red $(1.5 \mathrm{GHz})$ to blue $(1.0 \mathrm{GHz})$. The red contours show the hard X-ray source imaged by RHESSI. Panels (B) to (G) track one time-resolved type III burst. Panel (A) shows clearly that different bursts start at different frequencies and different positions, and that the radio sources follow different paths in the corona in different bursts. These paths do not correspond to any discernible structure in the EUV image. The authors compare the thermal electron density in the flux tubes carrying the electron beams, under the assumption of harmonic plasma emission, with the upper limit of the emission measure in the same regions determined by SDO/AIA. They conclude that individual electron beams propagated along bundles of magnetic field lines that were not larger than $100 \mathrm{~km}$. Chen et al. (2018) find such bundles to diverge from very compact regions, which they identify as reconnection regions around magnetic null points. Models of electron beam propagation in a fibrous corona were developed in the 1990s to understand the dynamic spectra of type III bursts (Roelof and Pick, 1989) or their relationship with type V continua (Raoult et al., 1990). Electron beams injected onto a bundle of field lines propagate or are absorbed depending on the characteristics of the beams, the local plasma conditions, and the magnetic field geometry (Raoult et al., 1990; Reid and Kontar, 2015), so that an apparently simple type III burst observed with low spectral and temporal resolution is resolved into numerous bursts by more powerful instruments. Multiple sources in individual type III burst groups were found by Pick and Ji (1986) at meter wavelengths, spanning a typical angular width of $25^{\circ}$ (see also Pick and van den Oord, 1990; Paesold et al., 2001; Ramesh et al., 2020). The new VLA observations allowed for the first time to map the sources in detail and to determine subtelescopic sizes involved in the propagation. The acceleration regions even in simple impulsive flares are multiple and connected to different parts of the high corona. This has bearing on the interpretation of measurements of energetic particles in space, for instance when modeling leads to the conclusion that different acceleration regions supply particles in a given impulsive SEP event, as in Kartavykh et al. (2007).

\subsection{Time-Extended and Delayed Acceleration of SEP Events}

Type III bursts have become a reference for the release of electrons, and charged particles in general, to the Heliosphere. Timing with respect to the onset of type III groups is used to distinguish different kinds of SEP events.

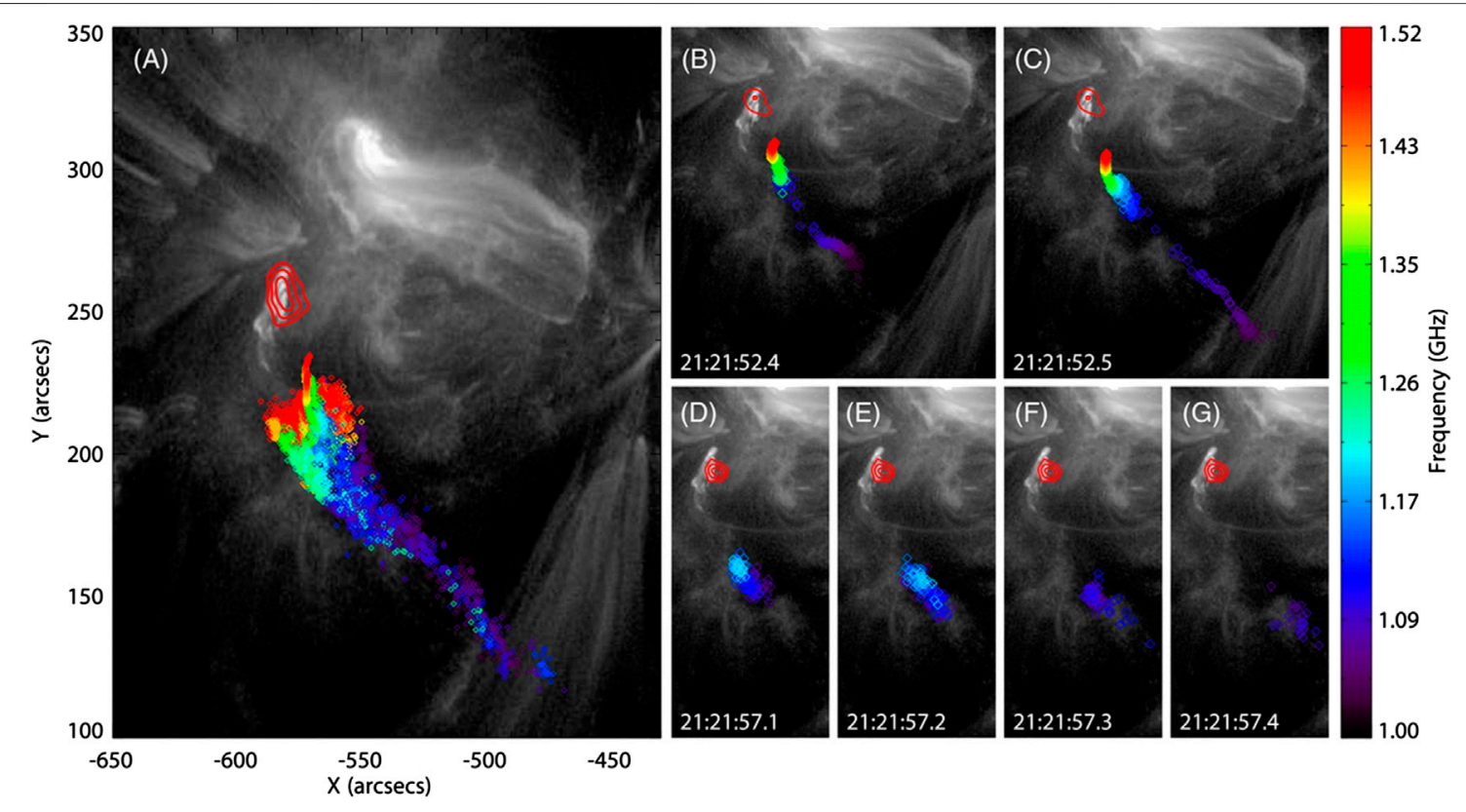

FIGURE 2 | Spectral imaging maps of a group of decimetric type III bursts (panel (A)) and an individual burst (B)-(G) with the VLA on November 5, 2011. The centroid positions of the radio source are coded in colors depending on the frequency as shown in the colorbar on the right, and overlaid on an SDO/AIA image at $13.1 \mathrm{~nm}$ wavelength. The red contours in (A) show the hard X-ray source mapped in the 12-25 keV range by RHESSI. From Chen et al. (2013). CAAS. Reproduced with permission. 


\subsubsection{Initial Solar Release Time of Anisotropic/Beamed Electron Events}

A detailed study of the initial electron release in simple impulsive events shows subtleties that are not captured by the simple picture of a single initial release time that is independent of energy and particle species. L. Wang and coworkers (Wang et al., 2006a; Wang et al., 2016) studied the release of electrons in impulsive 'scatter-free' events, where the time profile and the ordering of event onset with energy suggest a low rate of pitch-angle scattering. The interplanetary path length inferred from velocity dispersion analysis was found near $1.2 \mathrm{AU}$. These authors found a systematic ordering of release times, where electrons with energies below some limit near $10 \mathrm{keV}$ started to be released 1-30 min before the start of type III emission at $14 \mathrm{MHz}$, while electrons above $10 \mathrm{keV}(10-300 \mathrm{keV})$ tended to be released at or a few minutes $(0-17 \mathrm{~min})$ after the start of the type III bursts.

The energy of the electron beams emitting type III bursts is inferred indirectly. Identifying the energy of electrons arriving at the time when Langmuir waves started to be observed, Ergun et al. (1998) conclude that type III bursts at $\mathrm{km}$-wavelengths are emitted by electrons of 2-12 keV. Dulk et al. (1987) and Haggerty and Roelof (2006) derived similar typical values of $0.14 c$ and ranges of, respectively, $(0.07-0.25) c$ and $(0.06-0.35) c(0.9-34 \mathrm{keV})$ from the drift rates of the leading edge of type III bursts at frequencies below $1 \mathrm{MHz}$. Close to the Sun higher exciter speeds are usually estimated from the drift rates of type III bursts at meter wavelengths, by use of a density model for the ambient corona. Exciter speeds above $0.2 c$ are often quoted (see Sinclair Reid and Ratcliffe, 2014, and references therein). Reiner and MacDowall (2015) found a range $(0.2-0.38) c$ near the Sun, corresponding to energies in the range $(10-40) \mathrm{keV}$, and a lower exciter speed near $1 \mathrm{AU}$. The exciter speed is the speed where beam electrons resonate with Langmuir waves. The decrease from the corona to interplanetary space does not necessarily reveal an energy loss. Whatever the reason of the decrease, if the electrons producing type III emission close to the Sun are indeed more energetic than those at $1 \mathrm{AU}$, the electron timing by $\mathrm{L}$. Wang and coworkers is not inconsistent with events like May 1, 2000, where the start of type III bursts near $10 \mathrm{MHz}$ was found to coincide with the initial release of electrons above $20 \mathrm{keV}$ to the Heliosphere.

Krucker et al. (1999) and Haggerty and Roelof (2002) looked in detail into the onset timing of small and large electron events at energies up to some hundreds of $\mathrm{keV}$ observed, respectively, by Wind/3DP and ACE/EPAM. Events with a pronounced initial anisotropy were chosen, where one can expect that the onset time is closely related to the electron release at the Sun, rather than being determined by interplanetary propagation. Krucker and coworkers conducted a velocity-dispersion analysis of the onset of 58 events. The energy resolution of EPAM does not allow for such an analysis. Haggerty and Roelof (2002) assumed scatter-free propagation along a standard path length of $1.2 \mathrm{AU}$ to infer the solar release times for more than 100 events. Both papers report that the difference between the solar release times of the electrons and the start times of the type III bursts spans a broad range from zero to about $30 \mathrm{~min}$. Krucker et al. (1999) proposed that they could separate their 58 events into a sample of events with (42 cases) and without (16 cases) delay. The 16 events that were consistent with a common release with the type III bursts tended to have rather low energy, while the high-energy events were in the delayed sample. Results of the velocity-dispersion analysis of three events are shown in Figure 3. The vertical axis is the delay of the onset with respect to the start time of the type III emission at $14 \mathrm{MHz}$. The simultaneous release is illustrated by (A). The delayed sample comprised events where the release of electrons at all energies was delayed (B), as well as events where the low-energy electrons were released together with the first type III bursts, while the high-energy electrons were released later (C). The separation between 'low' and 'high' energy was around $25 \mathrm{keV}$, similar to Wang et al. (2016). The insets in the three panels show the positions of the parent flares of events in the same category as the plotted one with respect to the meridian $60^{\circ} \mathrm{W}$ and the ecliptic plane. Krucker et al. (1999) concluded that the events with no delay are small and well-connected, while the delayed events tend to be farther away from the nominal Parker spiral. In the November 6, 1997 event (Figure 3C) the parent flare was nominally well-connected in longitude, but not in latitude. Besides, it was observed a few hours before the arrival of an ICME (Wimmer-Schweingruber et al., 1999), i.e., in perturbed interplanetary conditions where the magnetic connection may not be adequately described by the Parker spiral field line. The red bars along the vertical axes in Figures 3B and 3C, which are not part of the original figure, show the approximate duration of the type III groups at $14 \mathrm{MHz}$. The delayed electron releases in the events in (B) and (C) started after the end of the type III bursts.

Delayed arrivals of particles near $1 \mathrm{AU}$ were also reported for protons (Krucker and Lin, 2000) and ions (Nitta et al., 2015; Wang et al., 2016) at $\mathrm{MeV} /$ nuc energies in simple impulsive events, with delays of order $1 \mathrm{~h}$, different from the May 1, 2000 event in Section 3.1.1. The uncertainties of the release times of $\mathrm{MeV} /$ nuc ions are rather large, however. Pick et al. (2006) report onset times, within an uncertainty of an hour, that are not inconsistent with a release in the vicinity of energetic electrons.

\subsubsection{Evidence on Time-Extended and Delayed Electron Release From Transport Modeling}

The interpretation of type III bursts as tracers of individual electron beams suggests to represent the injection function of SEP events as a series of impulsive releases. Agueda and coworkers used this approach to model short and long-lasting anisotropic electron events, in the same way as exposed above for the impulsive May 1, 2000 event. The particles are assumed to be released at a heliocentric distance of $2 R_{\odot}$ onto a Parker spiral field line, the shape of which is determined by the solar wind speed measured at the spacecraft. The release height is above the height inferred from radio observations in Section 3.1.2, but the difference amounts only to a few seconds for electrons traveling at about $c / 3$. Agueda et al. (2009) and Agueda et al. (2014) modeled the intensity and anisotropy of 17 electron events observed by ACE/EPAM and Wind/3DP at energies between about 30 and $300 \mathrm{keV}$, including impulsive and long-duration events. From the overall duration of the electron release they 

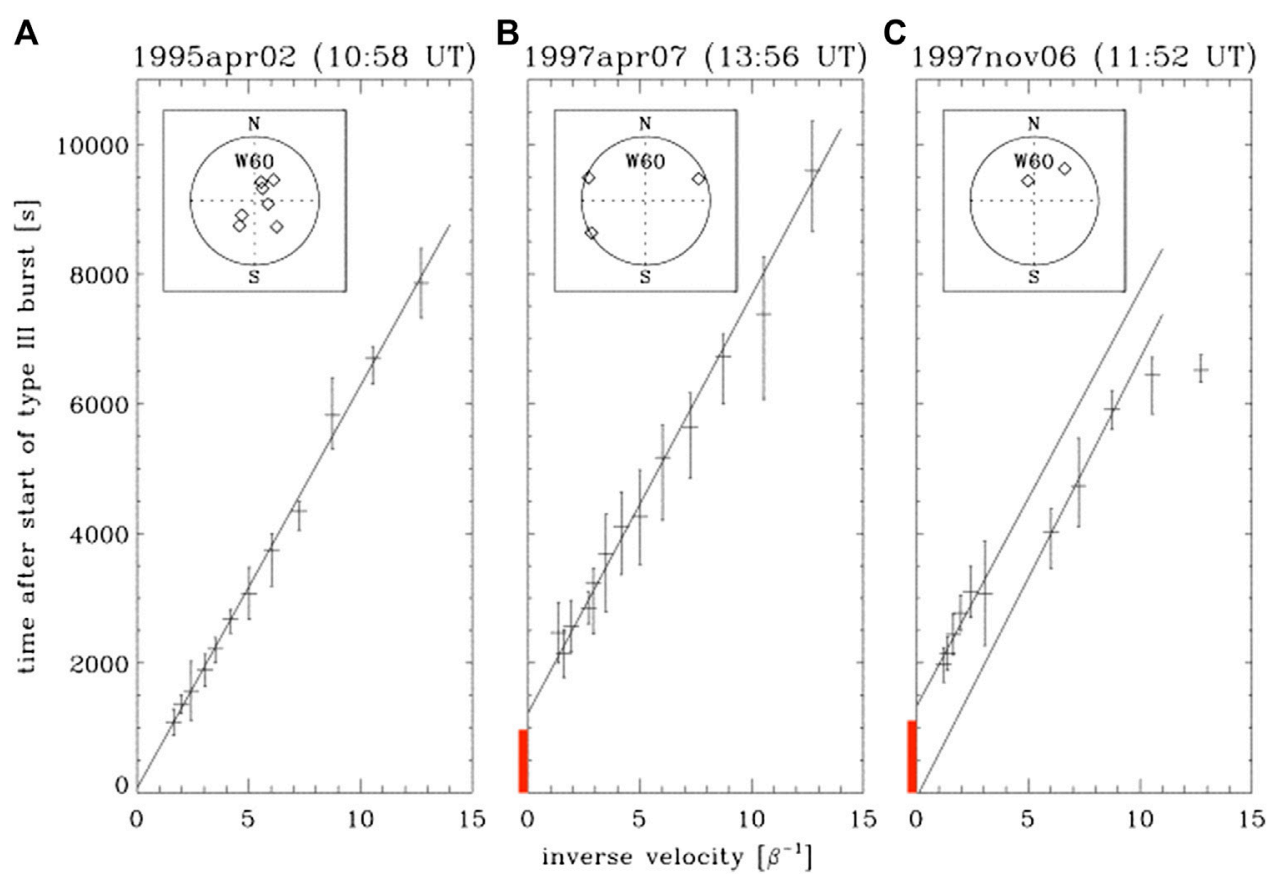

FIGURE 3 | Velocity dispersion analysis of three impulsive electron events showing different timing with respect to the start of the decametric type III bursts. The vertical axis shows the onset delay with respect to the start of the type III burst group: (A) simultaneous release of electrons at energies up to 80 keV; (B) delayed release of electrons at all energies; (C) simultaneous release of low-energy electrons below $\sim 30 \mathrm{keV}$, and delayed release of electrons at higher energies. The position of the associated flare is shown in the insets with respect to a position in the ecliptic plane at 60 W. From Krucker et al. (1999). (CAAS. Reproduced with permission. The red vertical bars in (B) and (C) are estimated durations of the type III burst groups, based on the dynamic spectra in Reiner et al. (2000).

distinguished short release episodes, lasting not longer than 15-30 min, and long episodes. Five events had only the short electron release at the time of the DH type III bursts. In six events the short initial release was followed, about $30 \mathrm{~min}$ later, by a delayed release lasting in general several hours, as on May 1, 2000. Six events were exclusively produced by a late release. Delayed releases were most often sustained in the sense that the individual impulses produced by the model were densely packed during several hours. Pacheco et al. (2019) applied the same type of analysis to HELIOS SEP observations as close to the Sun as 0.3 $\mathrm{AU}$ and found the same separation into short and long release episodes.

Agueda and coworkers noted that the short initial release intervals of electrons up to about $100 \mathrm{keV}$ coincided with hard X-ray emission and type III bursts in the solar atmosphere. In addition to the consistent timing, Agueda et al. (2009) found a correlation between the number of electrons released during this phase and the soft X-ray peak flux. They related these findings to a common acceleration of the interacting and escaping electrons at energies of tens to hundreds of keV during a flare. Similarly, James et al. (2017) found a correlation between spectral indices and numbers of electrons between 38 and at least $103 \mathrm{keV}$ measured near $1 \mathrm{AU}$ (ACE/EPAM) and observed through their hard X-ray emission in the chromosphere (RHESSI). They restricted the analysis to events with weak solar activity, where the soft X-ray emission was at most of importance C1.1 and no CME was observed (six events between 2004 and 2015). The ratio of escaping to interacting electrons ranged from
0.06 to 1.50 , assuming a uniform release of electrons into a cone of width $30^{\circ}$. The result differs from the analysis of more energetic events, where the numbers of electrons in space were found to be orders of magnitude below those inferred from the hard X-rays (Krucker et al., 2007).

Dröge and coworkers applied similar 1D models of electron transport to multi-spacecraft observations (Dresing et al., 2012; Dröge et al., 2014; Dröge et al., 2016). Again the start of the electron injection at the Sun (several tens of $\mathrm{keV}$ to $100 \mathrm{keV}$ ) was found at the time of the DH type III bursts. Delayed onsets were found when the initial rise of the observed intensity profiles had poorly observed anisotropy, and may therefore reveal transport effects (Dresing et al., 2012). The duration of the inferred injection differred between different vantage points in a given event. The different durations in fact reflect the different rise times seen from different vantage points. However, when using 3D models, allowing for transport across the heliospheric magnetic field, they reached agreement of the modeled time profiles of intensity and anisotropy with an injection that started with the DH III bursts and often had similar duration (Dröge et al., 2014; Dröge et al., 2016). The models have a number of free parameters that do not allow to consider this relationship as a firm proof, but they are the most detailed way to infer injection functions at the Sun. Their timing supports the idea that when electrons accelerated during flaring activity in the corona have access to space, the first electrons detected near $1 \mathrm{AU}$ are released with those emitting the $\mathrm{DH}$ type III bursts. Delays of the early arrival at the spacecraft can 
be due to a poor magnetic connection and the need of cross-field transport to reach the spacecraft.

Only the late, sustained electron release was observed in the large SEP event of December 26, 2001. As shown in the comparison between the modeled electron releases and the type III bursts observed below $14 \mathrm{MHz}$ in Figure 4A, ACE detected no electrons above $30 \mathrm{keV}$ from a release at the time of the type III bursts. The parent flare appeared well connected to the Earth $\left(54^{\circ} \mathrm{W}\right.$; Grechnev et al., 2017). However, Agueda et al. (2009) noted that the type III burst did not extend downward to the electron plasma frequency at the spacecraft, and explained this by electrons traveling along interplanetary field lines not connected to the spacecraft, as did Cane and Erickson (2003). The electron release to ACE coincided with the start of a type II radio burst. The soft X-ray and radio emissions during the same time interval as in Figure 4A are plotted in Figure 4B. The type II burst is clearly seen to originate at meter wavelengths, and to continue into the decameter wave range observed by Wind/ WAVES. It occurred at the low-frequency edge of a type IV continuum. The emission stands out in the flux density time histories at fixed frequencies up to $\mathrm{cm}$-wavelengths in the second panel from bottom. The type II and type IV radio emissions accompanied the entire time interval of electron release inferred from the transport model. They lasted clearly much longer than the microwave emission from the low corona. Grechnev and coworkers studied the complex evolution of this event in much detail (see Grechnev et al., 2017, and references therein). They identified a succession of two eruptions, and argued that the first, minor one, may have provided particularly favourable conditions for the acceleration of particles by the shock wave witnessed by the type II burst, and their escape to the Heliosphere. However, the type IV continuum is itself a signature of time-extended electron acceleration in the corona, related to the CME that drives the shock. A preferential association of late electron release with the type IV burst was reported in Laitinen et al. (2000).

The sustained late electron releases are in general not accompanied by type III bursts at decametric and longer wavelengths. This is a clear difference with respect to the impulsive flare phase. One possible reason (e.g., Raoult et al., 1990 ) is that a sustained electron release with a smooth time profile will create a bump-on-the tail distribution only at its start, whereafter the deficit of relatively low-energy electrons is continuously replenished. Similarly, the mutual overtaking of electron beams on the same field line will lead to the suppression of the radio emission (Briand et al., 2014). The absence of type III bursts during the sustained late releases suggests that the late acceleration is not just an extended version of the impulsive phase acceleration.

\subsubsection{Type III Bursts and the Initial Solar Release of Major SEP Events}

Type III bursts had for some time been considered as a characteristic counterpart of 'impulsive' SEP events (Reames, 1999), as discussed above, which are not particularly energetic.
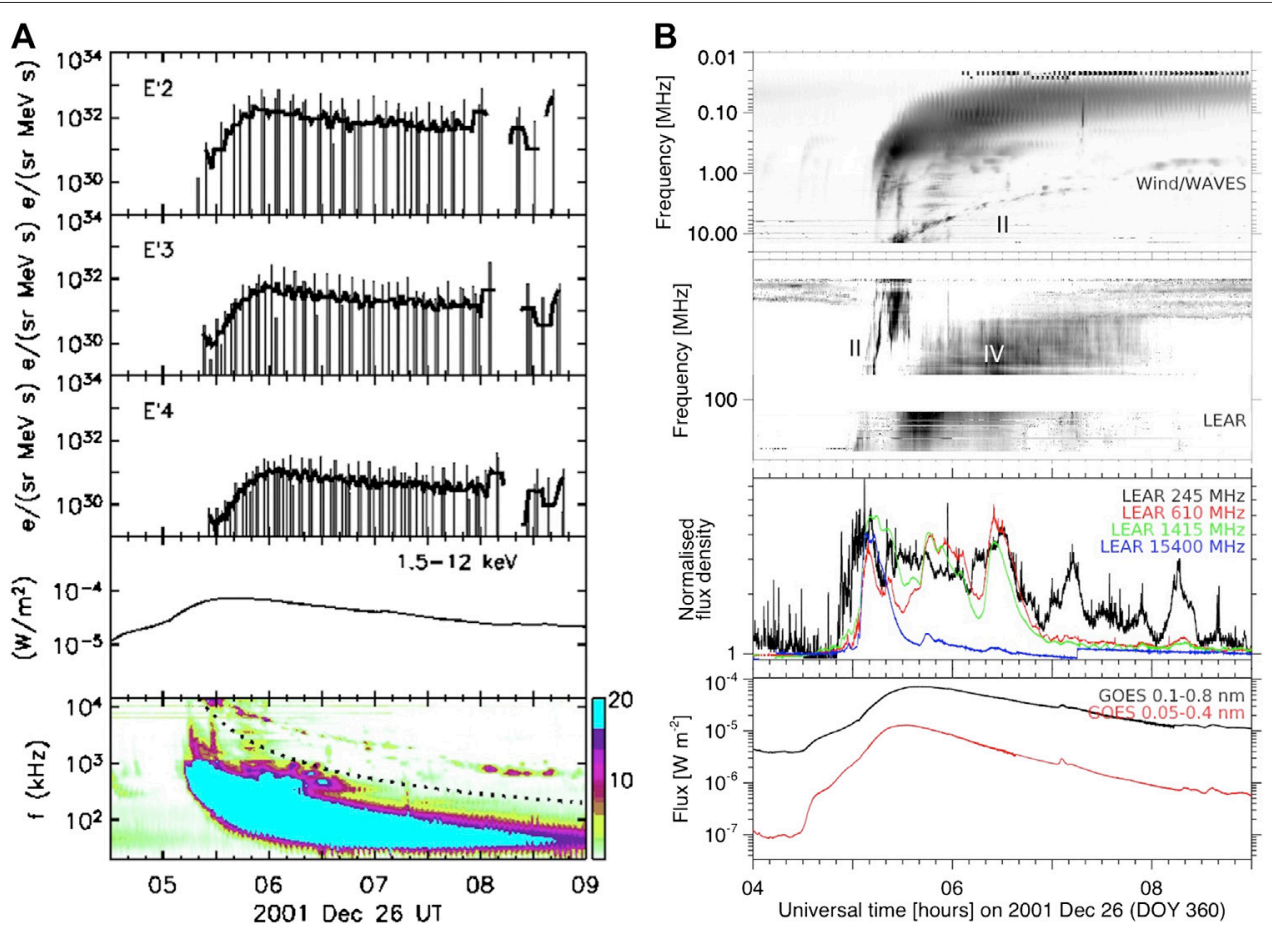

FIGURE 4 | Time histories of electron release, soft X-ray and radio emission during a large SEP event. (A) Injection delta-functions derived from the modeling of electrons in three energy channels of ACE/EPAM (three upper panels) compared with the soft X-ray flux (0.1-0.8 nm; second from bottom) and the dynamic radio spectrum between $14 \mathrm{MHz}$ and about $10 \mathrm{kHz}$ (bottom panel; vertical axis shows frequencies, increasing from bottom to top). From Agueda et al. (2009). ()ESO. Reproduced with permission. (B) Soft X-ray (bottom) and radio time histories (second from bottom), radio spectrum from $180 \mathrm{MHz}$ to $10 \mathrm{kHz}$ (frequency decreasing from bottom to top). Bursts of types II and IV are marked. Adapted from server. sepserver.eu 
Cane et al. (2002), however, demonstrated that groups of type III bursts accompany SEP events in general, occurring at the beginning of 121 of 123 SEP events they analyzed. Similarly, Cane et al. (2010) found that 278 out of 280 SEP events observed above $25 \mathrm{MeV} /$ nuc by IMP 8 and SoHO/ERNE were accompanied by type III bursts at dekametric and longer wavelengths. These type III emissions have a broad range of properties ranging from a few bursts that occur during the impulsive phase to large bright burst groups that continue well after the soft X-ray peak. Some other studies reported lower association rates, but all found similarly that most SEP events are accompanied by type III bursts (Vainio et al., 2013; Richardson et al., 2014; Kouloumvakos et al., 2015; Papaioannou et al., 2016; Miteva et al., 2017; Ameri et al., 2019). The histogram of durations of the burst groups at $14 \mathrm{MHz}$ (Cane et al., 2002) decreases monotonously from the lowest bin, 5-10 min, to the highest, $50-55 \mathrm{~min}$, with a median duration of about $20 \mathrm{~min}$. The large burst groups were initially termed 'shock-accelerated' or 'shock-associated' because of their frequent occurrence on the low-frequency side of type II bursts (Cane et al., 1981; Bougeret et al., 1998; Dulk et al., 2000). Cane et al. (2002) proposed the name type III- $l$ bursts, to capture long duration and low frequency in a single letter. Reiner and coworkers (Reiner et al., 2000) called them 'complex type III bursts'. In the following we consider type III bursts collectively. The peculiarities of the complex type III bursts are discussed in Section 2.4 of the companion chapter.

Cane et al. (2010) considered the timing of the DH type III bursts for different categories of SEP events. They showed that SEP events with a relatively high number of electrons (near $500 \mathrm{keV}$ ) relative to protons (near $25 \mathrm{MeV}$ ) tended to be accompanied by type III bursts during the impulsive phase. This sample comprised 80 out of 201 events that the authors could classify. The 2000 May 01 event is one of them. The events had relatively low intensity, corresponding to the common 'impulsive' SEP events. The other SEP events were accompanied by type III groups that tended to occur in the post-impulsive flare phase (cf. their Figure 13).

Several studies compared the initial release time of SEPs with the start time of the DH type III groups. The large uncertainties of the initial solar release time determination induce considerable uncertainty, with ensuing differences between different event catalogs that were discussed by Miteva et al. (2018). The high energy resolution of the SoHO/ERNE detector allows for a more detailed determination of the initial solar release of SEPs through the velocity-dispersion analysis. Several critical re-evaluations of the analysis methods were presented by Vainio et al. (2013), Kouloumvakos et al. (2015), and Ameri et al. (2019). Vainio et al. (2013) found that the initial release of deka-MeV protons occurred during groups of DH III bursts in $57 \%$ of the analyzed cases, and after the end of the type III bursts in $21 \%$, while Ameri et al. (2019) found fractions of $64 \%$ and $36 \%$, respectively. The first proton release was in the majority of the events delayed with respect to the start of the type III bursts. These studies did not consider the anisotropy of the SEPs.

\subsubsection{Delayed Onsets of Particle Events in Space: Possible Interpretations}

The delayed onset of SEP events in space can be explained by different processes including particle propagation in the Heliosphere, particle storage in the corona, and delayed acceleration at the Sun.

The storage of $\mathrm{MeV} / \mathrm{nuc}$ ions in the corona, already alluded to in Section 3.1.2, could explain delays, and the trapping times required to explain the observed charge states are not inconsistent with the reported onset delays. Wang et al. (2016) discard this interpretation, because it does not provide a common description of the escape of electrons and ions. Storage is hard to reconcile with the existence of type III bursts early in the event, and inconsistent with the fast escape of protons at $\mathrm{MeV}$ to deka$\mathrm{MeV}$ energies in events such as May 1, 2000.

Cane (2003) argued that the delayed onset of electron events could be explained by interplanetary transport. A possible process is enhanced pitch-angle scattering at energies above about $100 \mathrm{keV}$, which has been inferred, e.g., from the observed softening of electron spectra with increasing energy (Strauss et al., 2020). It is unclear if the energy dependence is sharp enough to explain a step-increase of the delay such as shown in Figure 3C. At the low-energy end of the electron spectrum, below a few tens of $\mathrm{keV}$, the energy loss due to the growth of Langmuir waves, which eventually leads to the type III bursts (Kontar and Reid, 2009; Reid and Kontar, 2013), implies that the electrons had higher energy during part of their travel than when they were detected. The expected flattening of the electron spectrum below a few tens of $\mathrm{keV}$ is sometimes observed (Krucker et al., 2007), but not always pronounced (Lin, 1985; Wang et al., 2016). These apparently low-energy electrons would then arrive earlier than expected, consistent with the observations by Wang et al. (2016). However, these authors discard interplanetary propagation as the cause of the delay, because the velocity-dispersion analysis of their events yielded a travel path comparable with a standard Parker spiral, which argues for a scatter-poor propagation at least of the first arriving particles.

Kahler and coworkers (Kahler, 2007; Kahler et al., 2007) envisaged a systematically larger travel path for electrons in delayed events, showing that the onset delays were anticorrelated with solar wind speed, and hence correlated with the length of the Parker spiral. But explaining delays of $10 \mathrm{~min}$ and more needs doubling the length of the Parker spiral. This cannot be a general interpretation, although the longitude of the travel path and the pitch angle scattering add to the uncertainties of the interpretation of the observed onset times (e.g., Kahler, 2007). New in situ measurements as a function of heliocentric distance will shed more light on the action of these processes.

Whenever the anisotropy of SEPs cannot be observed, the onset may be delayed by interplanetary transport. The possibility of transport across the average heliospheric magnetic field has come into focus again with the multi-spacecraft observations by STEREO. The mechanisms include particle drifts, the meandering of the heliospheric magnetic field lines due to motions of their solar footpoints, and wave-particle 
interactions (Dröge et al., 2010; Desai and Giacalone, 2016; Laitinen et al., 2016; Dalla et al., 2020).

However, the most common interpretation of onset delays is a delayed particle release at the Sun, not due to storage, but to a distinct acceleration process. Detailed comparisons of the electron spectra of delayed and prompt electron events corroborate the idea that the different onset times reveal different electron populations: Krucker et al. (2007) found that the prompt events had energy spectral slopes that correlated with those inferred from the hard X-ray emission of electrons in the solar atmosphere, while the spectra of delayed electron events showed a weaker correlation. A similar difference had been reported earlier between electron events of 'short' and 'long' duration, where Dröge (1996) compared rigidity spectra measured in situ between about 0.1 and $50 \mathrm{MeV}$ with the associated gamma-ray spectra in the range (0.3-1) MeV. Such differences do not exclude interplanetary transport effects, but are plausible if the prompt and short electron events in space result from a common acceleration with hard X-ray emitting electrons and with the electron beams emitting type III bursts, while the delayed long events come from a different acceleration process. The typical counterparts of sustained delayed particle releases are the long decay of the soft X-ray bursts, type II bursts at meter-todecameter wavelengths, type IV bursts at centimeter-to-meter wavelengths or a combination of these radio emissions. They are signatures of eruptive solar flares, where CMEs are additional sources of particle acceleration due to their shock waves and the processes of magnetic reconnection in their aftermath. In events where no prompt electron signature is seen, as in Figure 4, the interpretation implies that the prompt and delayed acceleration processes release particles onto different field lines, and that only those field lines guiding particles during the delayed release are connected to the spacecraft (cf. Klein et al., 2005).

\section{TYPE III BURSTS AS TRACERS OF PARTICLE PROPAGATION IN THE CORONA AND THE HELIOSPHERE}

\subsection{Confinement Versus Escape of Flare-accelerated Particles}

Type III bursts are a frequent manifestation of solar activity (Lin, 1985; Saint-Hilaire et al., 2013). This suggests that the processes of beam instabilities, growth of Langmuir waves, and conversion to escaping radio waves can operate easily, and that the absence of a type III burst conveys also significant information.

For instance, the absence of type III bursts allows one to identify confined flares, which are flares where the magnetic structure surrounding the region of energy release remains intact. Confined flares may be relatively strong: Wang and Zhang (2007) showed that about $10 \%$ of the X-class flares in solar cycle 23 were confined, since no CME was observed. Confined flares can be significant electron accelerators, as shown by the associated microwave or hard X-ray bursts (Schmahl et al., 1990; Gopalswamy et al., 1995; Klein et al., 2010; Thalmann et al., 2015). But there are no radio signatures of electrons escaping to the high corona (Gopalswamy et al., 2009; Klein et al., 2010). The GOES particle detectors recorded no excess above background, even when the flare was nominally well-connected to the Earth, although faint signatures may on occasion be detected by more sensitive instruments, as on SoHO and ACE (Klein et al., 2010). The absence of a significant SEP event has two reasons: the absence of a CME, which would have been an alternative source of particle acceleration, and the magnetic confinement of particles accelerated in the low corona, signaled by the absence of radio emission at metric and longer wavelengths.

Particle confinement may evolve during a solar eruptive event. Trottet et al. (1998) and Rieger et al. (1999) studied the radio emission at the times of two strong hard X-ray and gamma-ray flares. The initial hard X-ray and gamma-ray emission had no radio counterpart at frequencies below $1 \mathrm{GHz}$, and especially no type III emission. In the X15 flare on March 6, 1989 (Rieger et al., 1999) the gamma-rays in this initial phase of 2 min duration extended to above $20 \mathrm{MeV}$. A type II burst and a strong, long-lasting type IV continuum, indicative of the early CME evolution, appeared after the gamma-ray burst. In the event of Trottet et al. (1998), later gamma-ray peaks were accompanied by new radio sources, including type III emission. It appears from the successive appearance of gamma-ray peaks with new radio sources at dm$\mathrm{m}$ wavelengths that energy release and particle acceleration proceed stepwise from confined magnetic structures in the low corona (below $10^{5} \mathrm{~km}$, say) to larger spatial scales, higher in the corona, which then erupt into a CME (see also Chupp et al., 1993; Trottet et al., 1994; Akimov et al., 1996; Laitinen et al., 2000; Klein et al., 2014). The different steps proceed on time scales of tens of seconds to minutes. Radio signatures show that particles may escape from the flaring active region to the Heliosphere since the early onset of the acceleration, one to several minutes later, or not at all, depending on the evolution of the magnetic structure in a given event. Type III bursts at decametric and longer wavelengths are the clearest signatures of the earliest access of particles, not only electrons, to the Heliosphere during an eruptive solar flare.

\subsection{The Geometry of Open Magnetic Field Lines in the Corona}

When the existence of type III bursts demonstrates the escape of electrons to the high corona, imaging observations can identify those open field lines that are actually taken by the electron beams, and possibly by other species of charged particles. Parker's solar wind model implies that coronal regions around $50^{\circ} \mathrm{W}$ are best suited to make SEPs reach the Earth. Panels E, F of Figure 1 illustrate that the May 1, 2000 event is consistent with this expectation. The parent activity of simple impulsive SEP events in general clusters around this longitude (Reames, 1999; Nitta et al., 2006; Wang et al., 2012), albeit with some spread. One reason for this spread is that the Parker spiral as an average field line in interplanetary space makes sense only outside the solar wind source surface, while the geometry between the source surface and the low corona varies (Liewer et al., 2004; Wang et al., 2006b). This is illustrated by Figure 1D: open field lines are confined to a narrow region in the low atmosphere by the surrounding closed magnetic flux, but spread out at high altitudes, where the ambient closed flux is lacking, to cover a considerable fraction of the source surface. 
Particles accelerated in a flaring active region hence can have access to a wide range of longitudes and latitudes on the solar wind source surface. Klein et al. (2008) used radio images of type III bursts at meter wavelengths in eight impulsive SEP events where the initial solar release occurred during these type III bursts. The maps of type III sources showed that the electron beams propagate along coronal field lines that connect the parent active region to the vicinity of the nominal Parker spiral on the source surface even in a case where the parent active region was as far as $50^{\circ}$ away from the longitude of the footpoint of the nominal Parker spiral. Such a divergence of open magnetic field lines was inferred by Dulk et al. (1979) from the broadening of type III sources with decreasing frequency (see Mann et al., 2018, for an illustration using LOFAR). But the fact that the type III sources analyzed by Klein et al. (2008) are smaller than the bundle of open field lines rooted in the parent active region implies that electron beams are only injected onto a fraction, depending on the details of the acceleration region (e.g., Masson et al., 2012). Klassen et al. (2018) obtained a similar result from the analysis of an EUV jet related with an impulsive SEP event.

\subsection{The Geometry of the Heliospheric Magnetic Field}

Type III bursts at hectometric and kilometric wavelengths can in principle be used to infer the interplanetary trajectories of electron beams, and probe the geometry of field lines that guide particles through the Heliosphere. A number of techniques has been developed, which use the identification of the arrival direction of the radio waves alone or together with information on the density structure of the Heliosphere. The arrival direction can be determined by the modulation of the flux density recorded on a spinning spacecraft (Manning and Fainberg, 1980; Reiner and Stone, 1988, 1989), or by the autocorrelation and cross-correlation of signals recorded by antennas with different orientations on a three-axes stabilized spacecraft (Krupar et al., 2012). Krupar et al. (2020) validated their triangulation method with delay-time measurements of type III bursts between the STEREO A, Wind and Parker Solar Probe spacecraft. The results are found to be consistent with an error of $10-20 \%$.

In the case of type III storms observed during several successive days as the sources pass over the central meridian, an average trajectory can be defined. To the extent that one can assume a rigidly rotating source at a fixed heliocentric distance located in the ecliptic plane, the distance is determined by the time derivative of the measured elongation of the source. The azimuth is determined by the time of meridian passage at individual frequencies. Bougeret et al. (1984) showed that the sources of one such type III storm neatly aligned along a Parker spiral (Figure 5A).

Trajectories close to Parker spirals were also found for individual interplanetary type III bursts, often using triangulation from spacecraft at two or three vantage points
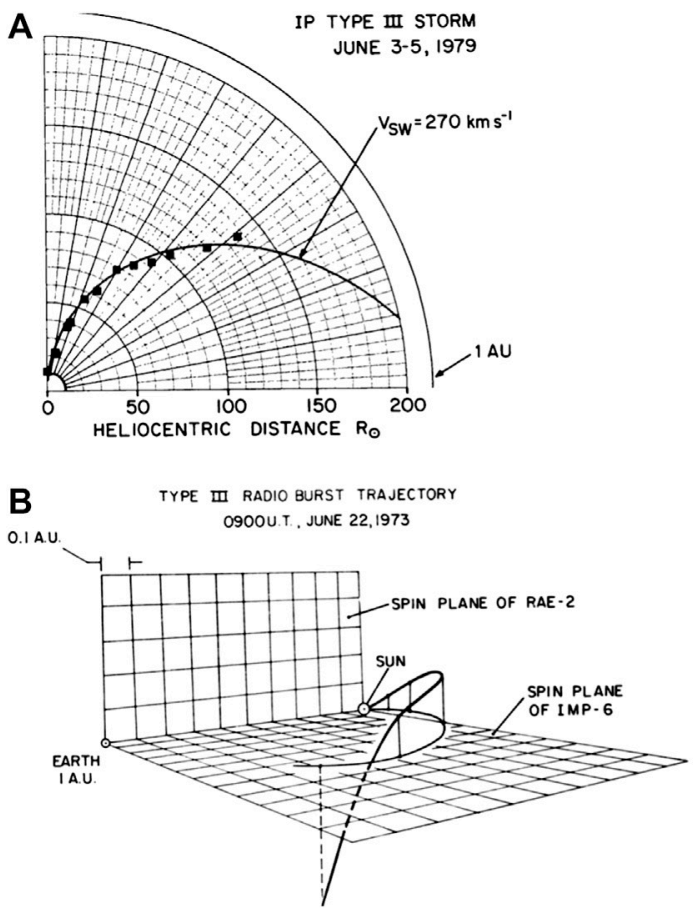

C

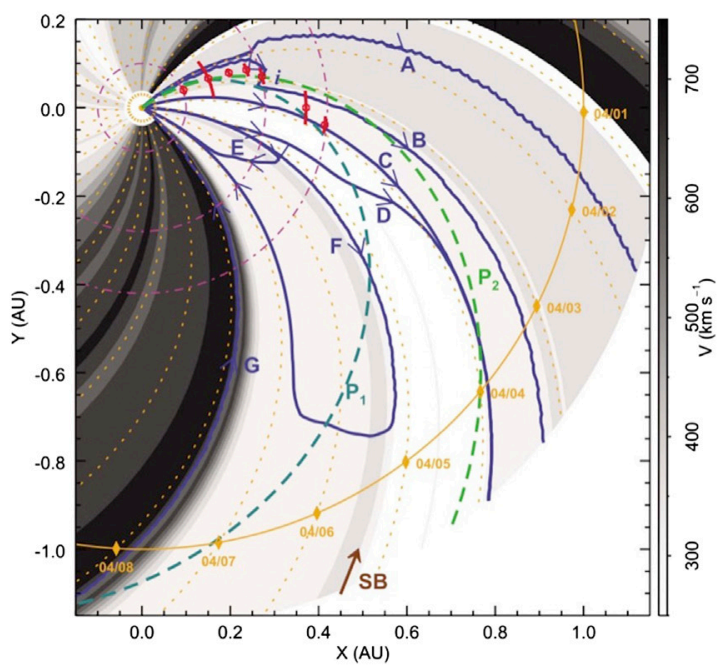

FIGURE 5 | Localization of interplanetary type III bursts; (A) Centroid positions of type III sources during a storm, with an overlaid Parker spiral field line for a solar wind speed of $270 \mathrm{~km} \mathrm{~s}^{-1}$ (Bougeret et al., 1984, (OESO). (B) 3D magnetic field line inferred from direction finding and a density model (Fitzenreiter et al., 1977 , figure from https://ntrs.nasa.gov/citations/19770005004). (C) Type III locations (red arcs, from Reiner et al., 2009) compared with two Parker spiral field lines $\left(P_{1}\right.$, $\left.P_{2}\right)$ as proposed by Reiner et al. and an alternative 2D magnetic field model (A)-(D), (F), from Li et al. (2016), @AGU. Figures reproduced with permission. 


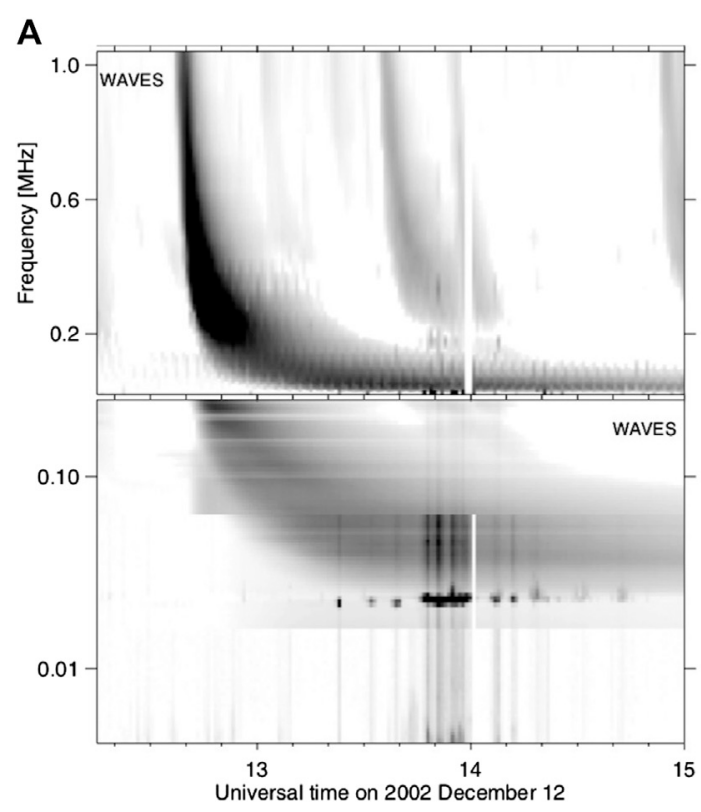

B

FIGURE 6 | Kilometric type III bursts and Langmuir waves as tracers of magnetic connection to the Sun on December 12, 2002: (A) Dynamic spectrum of radio emission between $1 \mathrm{MHz}$ and a few kHz; (B) Superposition of radio sources (iso-intensity contours at half maximum) at 164 and $237 \mathrm{MHz}$ on the EUV image (Klein and Posner, 2005). Sharply delimited rectangular gray areas and vertical bars on either side of the intense Langmuir waves in the spectra are instrumental artifacts. @ESO. Reproduced with permission.

(Fainberg and Stone, 1974; Weber et al., 1977; Reiner and Stone, 1986; Reiner et al., 1995; Reiner et al., 1998; Reiner et al., 2009; Martínez-Oliveros et al., 2012; Krupar et al., 2014). Lin et al. (1973) compared for two events the path lengths of electrons inferred from two methods: 1) the localization of type III bursts using direction finding and an interplanetary density model, 2) a velocity dispersion analysis (VDA) of electrons between about 6 and $50 \mathrm{keV}$. They found that the lengths of Parker spirals outlined by the type III sources, $1.25 \mathrm{AU}$ in both events, agreed well with those from VDA, 1.4 and 1.68 AU. The difference could be ascribed to the pitch angle scattering of electrons. The event with the longer path length from VDA had indeed a more diffusive time profile, and a lower drift rate of its type III emission than the other.

However, the application of different methods to the same events sometimes yielded substantially different results (Reiner et al., 2009; Martínez-Oliveros et al., 2012). A major source of uncertainty are the huge dimensions of the sources (see Reiner, 2001, and references therein), which makes the hypothesis uncertain that the lines of sight toward the type III source from two widely separated spacecraft point to the same target. Alternative models to a Parker spiral may be more consistent with the observations: Li et al. (2016) compared the positions of a type III burst measured by triangulations from the two STEREO and the Wind spacecraft by Reiner et al. (2009) with the geometry of a Parker spiral and the geometry predicted by a $2 \mathrm{D}$ model allowing for a finite azimuthal magnetic field component on the source surface. The comparison is displayed in Figure 5C. The Parker spiral $\mathrm{P}_{2}$ (green dashed line) is consistent with the type III burst locations, but intercepts a sector boundary. Li et al. (2016) conclude that in view of the actually observed interplanetary magnetic field configuration their alternative magnetic field model is more consistent with the type III burst trajectory than a Parker spiral.

Most models assume two-dimensional trajectories in the ecliptic plane, and some statistical studies support this hypothesis (Krupar et al., 2014). However, individual type III bursts may be better represented by $3 \mathrm{D}$ field lines. For instance, Fitzenreiter et al. (1977), combining direction finding from two spacecraft with a density model, inferred a trajectory at constant colatitude between the Sun and 0.3 AU, which then curves down to the ecliptic and crosses it at $0.8 \mathrm{AU}$ with an angle of $60^{\circ}$ (Figure 5B). Further evidence for field lines that rise out of the ecliptic plane near the Sun was provided by Dulk et al. (1986). Using observations from ISEE 3 around solar maximum (1980-81) they found that the latitude distribution of the radio sources near a heliocentric distance of $0.35-0.4$ AU increased from the ecliptic plane to a latitude of $10^{\circ}-15^{\circ}$, comparable to the average latitude of active regions at that time, and then decreased again. The authors concluded that field lines rooted in active regions often have constant latitude out to about $0.3 \mathrm{AU}$, as predicted by the Parker model, but bend at larger distance and become approximately parallel to the ecliptic plane. No detailed comparison seems to have been undertaken so far with models including a latitudinal component of the heliospheric magnetic field, which have been developed to account for unusual magnetic connections traced by energetic particles (see the reviews by Smith, 2008; Owens and Forsyth, 2013; Lhotka and Narita, 2019).

The Langmuir waves at the origin of type III bursts are only detectable when the spacecraft intercepts the source region, i.e., the electron beam (or one of the electron beams). This is a means to 
ascertain that the spacecraft is connected to the source region in the solar corona. On December 12, 2002 an impulsive SEP event occurred in association with a flare at N16 W36. The type III bursts are shown in the decametric-kilometric spectrum in Figure 6A. The radio positions at two metric wavelengths are overlaid as contours at half maximum on a nearly simultaneous EUV image in (B). Nitta et al. (2006) and Nitta and DeRosa (2008) noted that in this, like some other apparently simple SEP events, the potential field line extrapolations led to latitudes at the source surface with no connection to the ecliptic plane through a Parker spiral. The type III sources in Figure 6B confirm that in the corona the electron beams propagate along field lines that point out of the ecliptic plane, in agreement with the potential-field extrapolations. But Langmuir waves (bright short emissions in the lower panel of Figure 6A) accompanied the low-frequency part of the type III burst at the Wind spacecraft. They start at the time when the radio emission approaches the plasma frequency, which confirms that the Langmuir waves are generated by the electron beams producing the type III emission (Hoang et al., 1994). This implies in turn that the open magnetic field lines rooted in the parent active region turned down toward the ecliptic somewhere in the interplanetary medium. The interplanetary magnetic field must hence have had a significant latitudinal component.

Type III bursts seem to be unable to reveal finer details of the interplanetary magnetic field structure. Larson et al. (1997) and Kahler et al. (2011a,b) used the velocity dispersion of electron event onsets to probe the field line lengths in magnetic clouds. All studied electron events were accompanied by type III burst groups at decametric and longer wavelengths. However, the type III spectra do not seem to reflect even substantially different field line lengths. For example, the field line lengths were about $3 \mathrm{AU}$ for an electron event observed shortly after entry into a magnetic cloud, with a type III group at 19:56 UT on October 18, 1995, and about 1.2 AU at 10:28 UT the next day, near the axis of the magnetic cloud. Neither of the papers addresses the details of the type III spectra. Inspection using the $1 \mathrm{~min}$ data provided by NASA/GSFC ${ }^{2}$ shows that the type III burst with the longer travel path tends to show a slower drift at frequencies near and below $250 \mathrm{kHz}$ than the other one, but the difference is not pronounced and would not be a tool by itself to infer a longer field line. No obvious difference is seen between the type III bursts within and outside a magnetic cloud on 2004 August 30 and 31, respectively, both analyzed by Kahler et al. (2011b). This is probably related to the large sizes of the radio sources in the Heliosphere, usually ascribed to radio wave scattering, and to the possible mixture of fundamental and harmonic emission at a given radio frequency. Saturation of the radio receiver, which is seen in the rectangular brightenings in Figure $\mathbf{6 A}$ and the fact that an apparently single type III burst at decametric and longer wavelengths is composed of several individual bursts, which may be generated by electron beams on different magnetic field lines, could further confuse the picture. No detailed analysis has been published so far.

\footnotetext{
${ }^{2}$ https://cdaweb.gsfc.nasa.gov/pub/data/wind/waves/
}

Type III bursts are indicators of open magnetic field lines, but electron beams may also reveal closed field lines in the corona. In these cases upward-travelling electron beams create a type III-like burst drifting toward lower frequencies, but which turns over toward an opposite drift when the electron beams start to travel sunward after the apex of the loop. The result is a 'type U burst'. In the corona these bursts are well known to be rare phenomena (Aurass and Klein, 1997; Sinclair Reid and Ratcliffe, 2014; Reid and Kontar, 2017), even at decimetric wavelengths, which are emitted in the low corona where closed magnetic field structures dominate. Based on numerical simulations, Reid and Kontar argue especially that the small density gradient near the loop apex reduces the growth rate of Langmuir waves compared with the situation in an open flux tube. Another obvious reason is that the magnetic field divergence along open flux tubes acts to refocus a beam against pitch-angle diffusion, while during the downward propagation both processes combine to reduce the anisotropy. Leblanc et al. (1999) observed a type U burst with a turnover frequency at $1 \mathrm{MHz}$, at the time when a CME was observed with apex at $9 R_{\odot}$, consistent with plasma emission at the turnover frequency. The interpretation is the propagation of electron beams on the closed magnetic field lines of the CME. Démoulin et al. (2007) reported a burst that started as a type III burst, then turned to the opposite frequency drift, and again to a type III-like drift. The spectral signature resembles the letter $N$. The authors interpreted it as electron beams propagating along a switchback of a magnetic field line that had recently been formed by magnetic reconnection. But such observations of radio emission from sunward-streaming electrons are rare, and were only reported close to the Sun, at frequencies of $1 \mathrm{MHz}$ and above. No such signature has been observed with sunward-streaming electrons near 1 AU. Wang et al. (2011) analyzed an electron event with three successive enhancements at the Wind spacecraft, the first being due to a release of electron beams in the corona, associated with a type III burst, the second to electrons that were back-reflected at an obstacle outside $1 \mathrm{AU}$, and the third to anti-sunward electrons created by another reflection inside 1 AU. No reverse-drift type III bursts were observed with the second, sunward-propagating electron population. This can probably be explained by the fact that only relatively high-energy electrons $(>25 \mathrm{keV})$ were seen in the reflected electron populations, possibly because the low-energy electrons emitting type III emission in the interplanetary space are strongly focused, and therefore harder to reflect, while the angular distributions of electrons at energies above $15-20 \mathrm{keV}$ are broader (Lin, 1985). Similarly, Martínez Oliveros et al. (2020) analyzed an electron event where the Wind spacecraft observed electrons streaming back from the interplanetary space to the Sun, along the field lines of a magnetic cloud. The associated kilometric radio burst was of type III with no indication of a turnover.

\section{SUMMARY}

Radio bursts produced by non-thermal electrons in the corona are a unique tool to probe the acceleration and propagation of energetic particles. 
The radiative signatures in the low solar atmosphere of mildly relativistic electrons, i.e., hard X-ray bremsstrahlung and microwave gyrosynchrotron emission, have been shown to correlate well with nuclear gamma-ray emission and with different parameters of SEP events, including peak intensity and to some extent spectral hardness. This makes them useful for space weather purposes, especially since relevant data can be acquired with cheap equipment, while ground-based observations are well shielded against adverse space weather. But the interpretation is unclear, because all parameters of eruptive activity correlate with each other. This is especially the case of microwave peak flux and fluence, soft X-ray peak flux and fluence, and CME speed. The validity of statistical correlations therefore remains a subject of debate, with no obvious solution for the time being.

Type III radio bursts trace impulsive electron acceleration and their escape to the high corona and the Heliosphere. The achievement of a nearly seamless connection in radio spectrography combining observations from space and ground provides us with a tool to track electron propagation from the vicinity of the acceleration sites in the corona to the spacecraft. Type III bursts can therefore be used to establish or discard magnetic connections, which they trace in a more reliable way than the nominal interplanetary magnetic field models. The type III bursts show the acceleration regions are complex, and the propagation paths multiple. We have not yet succeeded to relate this to the very broad particle injections that multi-spacecraft measurements reveal. But the radio observations do show that the picture of a simple coronal acceleration region from which particles stream outward to space and downward to the chromosphere is only realized, if at all, in few very simple flares. This is consistent with theoretical expectations, as discussed by Vlahos et al. (2019).

The timing of SEPs gives clear evidence that in many events, especially large ones, electrons and protons are released over much longer durations than type III burst often with some delay. The most common interpretation is that a distinct delayed acceleration process is at work in

\section{REFERENCES}

Agueda, N., Klein, K.-L., Vilmer, N., Rodríguez-Gasén, R., Malandraki, O. E., Papaioannou, A., et al. (2014). Release timescales of solar energetic particles in the low corona. Astron. Astrophys. 570, A5. doi:10.1051/0004-6361/ 201423549

Agueda, N., Lario, D., Vainio, R., Sanahuja, B., Kilpua, E., and Pohjolainen, S. (2009). Modeling solar near-relativistic electron events. Insights into solar injection and interplanetary transport conditions. Astron. Astrophys. 507, 981-993. doi:10.1051/0004-6361/200912224

Agueda, N., Vainio, R., Lario, D., and Sanahuja, B. (2008). Injection and interplanetary transport of near-relativistic electrons: modeling the impulsive event on 2000 May 1. Astrophys. J. 675, 1601-1613. doi:10.1086/ 527527

Akimov, V. V., Ambrož, P., Belov, A. V., Berlicki, A., Chertok, I. M., Karlický, M., et al. (1996). Evidence for prolonged acceleration based on a detailed analysis of the long-duration solar gamma-ray flare of June 15, 1991. Sol. Phys. 166, 107-134. doi:10.1007/BF00179358 these events. This subject will be examined in the companion chapter.

\section{AUTHOR CONTRIBUTIONS}

The author confirms being the sole contributor of this work and has approved it for publication.

\section{FUNDING}

Centre National d'Etudes Spatiales (CNES) CNRS - INSU Observatoire de Paris Institut Polaire Paul-Emile Victor.

\section{ACKNOWLEDGMENTS}

This work owes much to the generous data provision of numerous space-borne and ground-based instruments and data bases, especially the SoHO/LASCO CME catalog generated and maintained at the CDAW Data Center by NASA and The Catholic University of America in cooperation with the Naval Research Laboratory, the Radio Monitoring web site at LESIA, Observatoire de Paris, supported by the French Space Agency CNES, the neutron monitor database maintained at the University of Kiel and built within a Framework 7 project of the European Union, and the National Centers for Environmental Information at NOAA. The author acknowledges helpful discussions with many colleagues during the NMDB, SEPSERver and HESPERIA projects funded by the European Union, during several working groups and workshops dedicated to solar energetic particles at the International Space Science Institute (ISSI) in Bern, and during workshops of the Community of European Solar Radio Astronomers (CESRA). He is indebted to the referees for their constructive criticism. This research was supported by the French Polar Institute (IPEV), the French space agency CNES, and the Programme National Soleil-Terre (PNST) of CNRS/INSU.

Akinyan, S. T., Mogilevsky, E. I., Böhme, A., and Krüger, A. (1971). Spectral features of large type IV bursts and interrelation to solar-terrestrial phenomena. Sol. Phys. 20, 112-121. doi:10.1007/BF00146102

Alissandrakis, C. E., Nindos, A., Patsourakos, S., Kontogeorgos, A., and Tsitsipis, P. (2015). A tiny event producing an interplanetary type III burst. Astron. Astrophys. 582, A52. doi:10.1051/0004-6361/201526265

Ameri, D., Valtonen, E., and Pohjolainen, S. (2019). Properties of high-energy solar particle events associated with solar radio emissions. Sol. Phys. 294, 122. doi:10. 1007/s11207-019-1512-9

Aschwanden, M. J. (2002). Particle acceleration and kinematics in solar flares. Space Sci. Rev. 101, 1-227. doi:10.1023/A:1019712124366

Aurass, H., and Klein, K.-L. (1997). Spectrographic and imaging observations of solar type U radio bursts. Astron. Astrophys. Suppl. 123, 279-304. doi:10.1051/aas:1997161

Avignon, Y., and Pick-Gutmann, M. (1959). Relation entre les émissions solaires de rayons cosmiques et les sursauts de type IV. Acad. des Sci. Paris Comptes Rendus 249, 2276-2278.

Balch, C. C. (2008). Updated verification of the Space Weather Prediction Center's solar energetic particle prediction model. Space Weather 6, S01001. doi:10.1029/ 2007SW000337 
Boischot, A., and Denisse, J.-F. (1957). Les émissions de type IV et l'origine des rayons cosmiques associés aux éruptions chromosphériques. Acad. des Sci. Paris Comptes Rendus. 245, 2194-2197.

Boischot, A., and Warwick, J. W. (1959). Radio emission following the flare of August 22, 1958. J. Geophys. Res. 64, 683-684. doi:10.1029/JZ064i006p00683

Bougeret, J.-L., Zarka, P., Caroubalos, C., Karlický, M., Leblanc, Y., Maroulis, D., et al. (1998). A shock-associated (SA) radio event and related phenomena observed from the base of the solar corona to 1 AU. Geophys. Res. Lett. 25, 2513-2516. doi:10.1029/98GL50563

Bougeret, J. L., Fainberg, J., and Stone, R. G. (1984). Interplanetary radio storms. II emission levels and solar wind speed in the range 0.05-0.8 AU. Astron. Astrophys. 141, 17-24.

Briand, C., Henri, P., and Hoang, S. (2014). Inhibition of type III radio emissions due to the interaction between two electron beams: observations and simulations. J. Geophys. Res. 119, 2365-2378. doi:10.1002/2013JA019688

Cane, H. V., and Erickson, W. C. (2003). Energetic particle propagation in the inner heliosphere as deduced from low-frequency $(<100 \mathrm{kHz})$ observations of type III radio bursts. J. Geophys. Res. 108, 1203. doi:10.1029/2002JA009488

Cane, H. V., Erickson, W. C., and Prestage, N. P. (2002). Solar flares, type III radio bursts, coronal mass ejections and energetic particles. J. Geophys. Res. 107, 1315. doi:10.1029/2001JA000320

Cane, H. V. (2003). Near-relativistic solar electrons and type III radio bursts. Astrophys. J. 598, 1403-1408. doi:10.1086/379007

Cane, H. V., Richardson, I. G., and von Rosenvinge, T. T. (2010). A study of solar energetic particle events of 1997-2006: their composition and associations. J. Geophys. Res. 115, A08101. doi:10.1029/2009JA014848

Cane, H. V., Stone, R. G., Fainberg, J., Steinberg, J. L., Hoang, S., and Stewart, R. T. (1981). Radio evidence for shock acceleration of electrons in the solar corona. Geophys. Res. Lett. 8, 1285-1288. doi:10.1029/GL008i012p01285

Carley, E. P., Vilmer, N., and Vourlidas, A. (2020). Radio observations of coronal mass ejection initiation and development in the low solar corona. Front. Astron. Space Sci. 7, 551558. doi:10.3389/fspas.2020.551558

Castelli, J. P., Aarons, J., and Michael, G. A. (1967). Flux density measurements of radio bursts of proton-producing flares and nonproton flares. J. Geophys. Res. 72, 5491-5498. doi:10.1029/JZ072i021p05491

Castelli, J. P., and Barron, W. R. (1977). A catalog of solar radio bursts 1966-1976 having spectral characteristics predictive of proton activity. J. Geophys. Res. 82, 1275-1278. doi:10.1029/JA082i007p01275

Chen, B., Bastian, T. S., White, S. M., Gary, D. E., Perley, R., Rupen, M., et al. (2013). Tracing electron beams in the Sun's corona with radio dynamic imaging spectroscopy. Astrophys. J. Lett. 763, L21. doi:10.1088/2041-8205/ $763 / 1 / \mathrm{L} 21$

Chen, B., Yu, S., Battaglia, M., Farid, S., Savcheva, A., Reeves, K. K., et al. (2018). Magnetic reconnection null points as the origin of semirelativistic electron beams in a solar jet. Astrophys. J. 866, 62. doi:10.3847/1538-4357/aadb89

Chertok, I. M. (2018). Diagnostic analysis of the solar proton flares of September 2017 by their radio bursts. Geomagn. Aeron. 58, 457-463. doi:10.1134/ S0016793218040035

Chertok, I. M., Grechnev, V. V., and Meshalkina, N. S. (2009). On the correlation between spectra of solar microwave bursts and proton fluxes near the Earth. Astron. Rep. 53, 1059-1069. doi:10.1134/S1063772909110110

Chertok, I. M. (1990). On the correlation between the solar gamma-ray line emission, radio bursts and proton fluxes in the interplanetary space. Astron. Nachr. 311, 379-381. doi:10.1002/asna.2113110618

Chupp, E. L., Trottet, G., Marschhauser, H., Pick, M., Soru-Escaut, L., Rieger, E., et al. (1993). A study of the evolution of electron and ion acceleration during the 09:09 UT solar flare on 1989 September 9. Astron. Astrophys. 275, 602.

Cliver, E., and Kahler, S. (1991). High coronal flares and impulsive acceleration of solar energetic particles. Astrophys. J. 366, L91-L94.

Cliver, E. W. (2016). Flare vs. shock acceleration of high-energy protons in solar energetic particle events. Astrophys. J. 832, 128. doi:10.3847/0004-637X/832/ $2 / 128$

Correia, E., Kaufmann, P., and Magun, A. (1994). “The observed spectrum of solar burst continuum emission in the submillimeter spectral range," in IAU symp. 154: infrared solar Physics. Editors D. Rabin, J. Jefferies, and C. Lindsey. Dordrecht, Netherlands: Springer, 125-129.

Croom, D. L. (1971). Solar microwave bursts as indicators of the occurrence of solar proton emission. Sol. Phys. 19, 152-170. doi:10.1007/BF00148831
Daibog, E. I., Mel'Nikov, V. F., and Stolpovskii, V. G. (1993). Solar energetic particle events from solar flares with weak impulsive phases of microwave emission. Sol. Phys. 144, 361-372. doi:10.1007/BF00627600

Daibog, E. I., Stolpovskii, V. G., Melnikov, V. F., and Podstrigach, T. S. (1989). Microwave bursts and the relative abundance of electrons and protons in cosmic-rays from solar flares. Sov. Astron. Lett. 15, 432-436.

Dalla, S., de Nolfo, G. A., Bruno, A., Giacalone, J., Laitinen, T., Thomas, S., et al. (2020). 3D propagation of relativistic solar protons through interplanetary space. Astron. Astrophys. 639, A105. doi:10.1051/0004-6361/201937338

Démoulin, P., Klein, K.-L., Goff, C. P., van Driel-Gesztelyi, L., Culhane, J. L., Mandrini, C. H., et al. (2007). Decametric N burst: a consequence of the interaction of two coronal mass ejections. Sol. Phys. 240, 301-313. doi:10.1007/ s11207-006-0259-2

Desai, M., and Giacalone, J. (2016). Large gradual solar energetic particle events. Living Rev. Sol. Phys. 13, 3. doi:10.1007/s41116-016-0002-5

Dierckxsens, M., Tziotziou, K., Dalla, S., Patsou, I., Marsh, M. S., Crosby, N. B., et al. (2015). Relationship between solar energetic particles and properties of flares and CMEs: statistical analysis of solar cycle 23 events. Sol. Phys. 290, 841-874. doi:10.1007/s11207-014-0641-4

DiFabio, R., Guo, Z., Möbius, E., Klecker, B., Kucharek, H., Mason, G. M., et al. (2008). Energy-dependent charge states and their connection with ion abundances in impulsive solar energetic particle events. Astrophys. J. 687, 623-634. doi:10.1086/591833

Dresing, N., Gómez-Herrero, R., Klassen, A., Heber, B., Kartavykh, Y., and Dröge, W. (2012). The large longitudinal spread of solar energetic particles during the 17 January 2010 solar event. Sol. Phys. 281, 281-300. doi:10.1007/s11207-0120049-y

Dröge, W. (1996). "Energetic solar electron spectra and gamma-ray observations," in High energy solar Physics, Greenbelt, MD, August 16-18, 1995. Editors R. Ramaty, N. Mandzhavidze, and X.-M. Hua (Woodbury, NY: American Institute of Physics), 78-85.

Dröge, W., Kartavykh, Y. Y., Dresing, N., Heber, B., and Klassen, A. (2014). Wide longitudinal distribution of interplanetary electrons following the 7 February 2010 solar event: observations and transport modeling. J. Geophys. Res. 119, 6074-6094. doi:10.1002/2014JA019933

Dröge, W., Kartavykh, Y. Y., Dresing, N., and Klassen, A. (2016). Multi-spacecraft observations and transport modeling of energetic electrons for a series of solar particle events in August 2010. Astrophys. J. 826, 134. doi:10.3847/0004-637X/ $826 / 2 / 134$

Dröge, W., Kartavykh, Y. Y., Klecker, B., and Kovaltsov, G. A. (2010). Anisotropic three-dimensional focused transport of solar energetic particles in the inner heliosphere. Astrophys. J. 709, 912-919. doi:10.1088/0004-637X/709/2/912

Dulk, G. A., Goldman, M. V., Steinberg, J. L., and Hoang, S. (1987). The speeds of electrons that excite solar radio bursts of type III. Astron. Astrophys. 173, 366-374.

Dulk, G. A., Leblanc, Y., Bastian, T. S., and Bougeret, J. L. (2000). Acceleration of electrons at type II shock fronts and production of shock-accelerated type III bursts. J. Geophys. Res. 105, 27343-27352.

Dulk, G. A., Melrose, D. B., and Suzuki, S. (1979). Evidence for extreme divergence of open field lines from solar active regions. Proc. Astron. Soc. Aust. 3, 375-379.

Dulk, G. A., Steinberg, J. L., Hoang, S., and Lecacheux, A. (1986). "Latitude distribution of interplanetary magnetic field lines rooted in active regions," in The sun and the heliosphere in three dimensions. Editor R. G. Marsden (Dordrecht, Netherlands: Springer), 229-233.

Ergun, R. E., Larson, D., Lin, R. P., McFadden, J. P., Carlson, C. W., Anderson, K. A., et al. (1998). Wind spacecraft observations of solar impulsive electron events associated with solar type III radio bursts. Astrophys. J. 503, 435-445. doi:10. $1086 / 305954$

Fainberg, J., and Stone, R. G. (1974). Satellite observations of type III solar radio bursts at low frequencies. Space Sci. Rev. 16, 145-188. doi:10.1007/BF00240885

Fitzenreiter, R. J., Fainberg, J., Weber, R. R., Alvarez, H., Haddock, F. T., and Potter, W. H. (1977). Radio observations of interplanetary magnetic field structures out of the ecliptic. Sol. Phys. 52, 477-484. doi:10.1007/BF00149662

Fletcher, L., Dennis, B. R., Hudson, H. S., Krucker, S., Phillips, K., Veronig, A., et al. (2011). An observational overview of solar flares. Space Sci. Rev. 159, 19-106. doi:10.1007/s11214-010-9701-8

Fomichev, V. V., and Chertok, I. M. (1985). Relation between gamma-ray emission, radio bursts, and proton fluxes from solar flares. Sov. Astron. 29, 554-559. 
Forrest, D. J., and Chupp, E. L. (1983). Simultaneous acceleration of electrons and ions in solar flares. Nature. 305, 291-292. doi:10.1038/305291a0

Gopalswamy, N., Akiyama, S., and Yashiro, S. (2009). "Major solar flares without coronal mass ejections," in Universal heliophysical processes. Editors N. Gopalswamy and D. F. Webb (Paris, France: International Astronomical Union), 283-286. doi:10.1017/S174392130902941X

Gopalswamy, N. (2009). "Coronal mass ejections and space weather," in Climate and weather of the sun-earth system (CAWSES) selected papers from the 2007 kyoto symposium. Editors T. Tsuda, R. Fujii, K. Shibata, and M. A. Geller (Tokyo, Japan: TERRAPUB), 77-120.

Gopalswamy, N., Raulin, J.-P., Kundu, M. R., Nitta, N., Lemen, J. R., Herrmann, R., et al. (1995). VLA and YOHKOH observations of an M1.5 flare. Astrophys. J. 455, 715-732. doi:10.1086/176618

Grechnev, V., Uralov, A. M., Kiselev, V. I., and Kochanov, A. A. (2017). The 26 December 2001 solar eruptive event responsible for GLE63. II. Multi-loop structure of microwave sources in a major long-duration flare. Sol. Phys. 292, 3. doi:10.1007/s11207-016-1025-8

Grechnev, V. V., Kiselev, V. I., Meshalkina, N. S., and Chertok, I. M. (2015). Relations between microwave bursts and near-Earth high-energy proton enhancements and their origin. Sol. Phys. 290, 2827-2855. doi:10.1007/s11207-015-0797-6

Grechnev, V. V., Meshalkina, N. S., Chertok, I. M., and Kiselev, V. I. (2013). Relations between strong high-frequency microwave bursts and proton events. Publ. Astron. Soc. Jpn. 65, 4. doi:10.1093/pasj/65.sp1.S4

Haggerty, D. K., and Roelof, E. C. (2002). Impulsive near-relativistic solar electron events: delayed injection with respect to solar electromagnetic emission. Astrophys. J. 579, 841-853. doi:10.1086/342870

Haggerty, D. K., and Roelof, E. C. (2006). Leading edge and peak flux density exciter speeds for well connected type-III bursts. Adv. Space Res. 38, 1001-1006. doi:10.1016/j.asr.2005.09.035

Hakura, Y., and Goh, T. (1959). Pre-SC polar cap ionospheric blackout and type IV solar radio outburst. J. Radio Res. Lab. 6, 635-650.

Hoang, S., Dulk, G., and Leblanc, Y. (1994). Interplanetary type III radio bursts that approach the plasma frequency: ulysses observations. Astron. Astrophys. 289, 957-971.

Hurford, G. J., Krucker, S., Lin, R. P., Schwartz, R. A., Share, G. H., and Smith, D. M. (2006). Gamma-ray imaging of the 2003 October/November solar flares. Astrophys. J. Lett. 644, L93-L96. doi:10.1086/505329

Isaeva, E. A., Melnikov, V. F., and Tsvetkov, L. I. (2010). Dependence of the SCR proton flux estimate on radio burst parameters. Bull. Crime. Astrophys. Obs. 106, 26-30. doi:10.3103/S0190271710010043

James, T., Subramanian, P., and Kontar, E. P. (2017). Small electron acceleration episodes in the solar corona. Mon. Not. Roy. Astron. Soc. 471, 89-99. doi:10. 1093/mnras/stx1460

Kahler, S., Reames, D., and Sheeley, N. (2001). Coronal mass ejections associated with impulsive solar energetic particle events. Astrophys. J. 562, 558-565. doi:10. $1086 / 323847$

Kahler, S. W., Aurass, H., Mann, G., and Klassen, A. (2007). Solar radio burst and solar wind associations with inferred near-relativistic electron injections. Astrophys. J. 656, 567-576. doi:10.1086/510230

Kahler, S. W., Haggerty, D. K., and Richardson, I. G. (2011a). Magnetic field-line lengths in interplanetary coronal mass ejections inferred from energetic electron events. Astrophys. J. 736, 106. doi:10.1088/0004-637X/736/2/106

Kahler, S. W., Krucker, S., and Szabo, A. (2011b). Solar energetic electron probes of magnetic cloud field line lengths. J. Geophys. Res. 116, A01104. doi:10.1029/ 2010JA015328

Kahler, S. W. (1982a). Radio burst characteristics of solar proton flares. Astrophys. J. 261, 710-719. doi:10.1086/160381

Kahler, S. W. (1982b). The role of the big flare syndrome in correlations of solar energetic proton fluxes and associated microwave burst parameters. J. Geophys. Res. 87, 3439-3448. doi:10.1029/JA087iA05p03439

Kahler, S. W. (2007). Solar sources of heliospheric energetic electron events shocks or flares? Space Sci. Rev. 129, 359-390. doi:10.1007/s11214-007-9143-0

Kane, S. R., Chupp, E. L., Forrest, D. J., Share, G. H., and Rieger, E. (1986). Rapid acceleration of energetic particles in the 1982 February 8 solar flare. Astrophys. J. Lett. 300, L95-L98. doi:10.1086/184610

Kartavykh, Y. Y., Dröge, W., Klecker, B., Mason, G. M., Möbius, E., Popecki, M., et al. (2007). Evidence of a two-temperature source region in the ${ }^{3} \mathrm{He}$-rich solar energetic particle event of 2000 May 1. Astrophys. J. 671, 947-954. doi:10.1086/522687
Kiener, J., Gros, M., Tatischeff, V., and Weidenspointner, G. (2006). Properties of the energetic particle distributions during the October 28, 2003 solar flare from INTEGRAL/SPI observations. Astron. Astrophys. 445, 725-733. doi:10.1051/ 0004-6361:20053665

Klassen, A., Dresing, N., Gómez-Herrero, R., Heber, B., and Veronig, A. (2018). Strong non-radial propagation of energetic electrons in solar corona. Astron. Astrophys. 614, A61. doi:10.1051/0004-6361/201732041

Klecker, B., Kunow, H., Cane, H. V., Dalla, S., Heber, B., Kecskemety, K., et al. (2006). Energetic particle observations. Space Sci. Rev. 123, 217-250. doi:10. 1007/s11214-006-9018-9

Klecker, B., Möbius, E., and Popecki, M. A. (2007). Ionic charge states of solar energetic particles: a clue to the source. Space Sci. Rev. 130, 273-282. doi:10. 1007/s11214-007-9207-1

Klein, K.-L., Aurass, H., Soru-Escaut, I., and Kalman, B. (1997). Electron acceleration sites in a large-scale coronal structure. Astron. Astrophys. 320, 612-619.

Klein, K.-L., and Dalla, S. (2017). Acceleration and propagation of solar energetic particles. Space Sci. Rev. 212, 1107-1136. doi:10.1007/s11214-017-0382-4

Klein, K.-L., Krucker, S., Lointier, G., and Kerdraon, A. (2008). Open magnetic flux tubes in the corona and the transport of solar energetic particles. Astron. Astrophys. 486, 589-596. doi:10.1051/0004-6361:20079228

Klein, K.-L., Krucker, S., Trottet, G., and Hoang, S. (2005). Coronal phenomena at the onset of solar energetic electron events. Astron. Astrophys. 431, 1047-1060. doi:10.1051/0004-6361:20041258

Klein, K.-L., Masson, S., Bouratzis, C., Grechnev, V., Hillaris, A., and PrekaPapadema, P. (2014). The relativistic solar particle event of 2005 January 20: origin of delayed particle acceleration. Astron. Astrophys. 572, A4. doi:10.1051/ 0004-6361/201423783

Klein, K.-L., and Posner, A. (2005). The onset of solar energetic particle events: prompt release of deka-MeV protons and associated coronal activity. Astron. Astrophys. 438, 1029-1042. doi:10.1051/0004-6361:20042607

Klein, K.-L. (2021). Radio astronomical tools for the study of solar energetic particles II. Time-extended acceleration at subrelativistic and relativistic energies. Front. Astron. Space Sci. doi:10.3389/fspas.2020.580445

Klein, K.-L., Trottet, G., and Klassen, A. (2010). Energetic particle acceleration and propagation in strong CME-less flares. Sol. Phys. 263, 185-208. doi:10.1007/ s11207-010-9540-5

Knipp, D. (2011). Understanding space weather and the physics behind it. New York, NY: McGraw-Hill. doi:10.1007/978-3-319-43495-7

Kocharov, L. G., Kovaltsov, G. A., and Torsti, J. (2001). Dynamical cycles in charge and energy for iron ions accelerated in a hot plasma. Astrophys. J. 556, 919-927. doi:10.1086/321596

Kocharov, L., Kovaltsov, G. A., Torsti, J., and Ostryakov, V. M. (2000). Evaluation of solar energetic Fe charge states: effect of proton-impact ionization. Astron. Astrophys. 357, 716-724.

Kontar, E. P., and Reid, H. A. S. (2009). Onsets and spectra of impulsive solar energetic electron events observed near the Earth. Astrophys. J. Lett. 695, L140-L144. doi:10.1088/0004-637X/695/2/L140

Kouloumvakos, A., Nindos, A., Valtonen, E., Alissandrakis, C. E., Malandraki, O., Tsitsipis, P., et al. (2015). Properties of solar energetic particle events inferred from their associated radio emission. Astron. Astrophys. 580, A80. doi:10.1051/ 0004-6361/201424397

Krucker, S., Kontar, E. P., Christe, S., and Lin, R. P. (2007). Solar flare electron spectra at the Sun and near the Earth. Astrophys. J. Lett. 663, L109-L112. doi:10. $1086 / 519373$

Krucker, S., Larson, D. E., Lin, R. P., and Thompson, B. J. (1999). On the origin of impulsive electron events observed at 1 AU. Astrophys. J. 519, 864-875. doi:10. $1086 / 307415$

Krucker, S., and Lin, R. P. (2000). Two classes of solar proton events derived from onset time analysis. Astrophys. J. 542, L61-L64. doi:10.1086/312922

Krupar, V., Maksimovic, M., Santolik, O., Cecconi, B., and Kruparova, O. (2014). Statistical survey of type III radio bursts at long wavelengths observed by the solar terrestrial relations observatory (STEREO)/waves instruments: goniopolarimetric properties and radio source locations. Sol. Phys. 289, 4633-4652. doi:10.1007/s11207-014-0601-z

Krupar, V., Santolik, O., Cecconi, B., Maksimovic, M., Bonnin, X., Panchenko, M., et al. (2012). Goniopolarimetric inversion using SVD: an application to type III radio bursts observed by STEREO. J. Geophys. Res. 117, A06101. doi:10.1029/ 2011JA017333 
Krupar, V., Szabo, A., Maksimovic, M., Kruparova, O., Kontar, E. P., Balmaceda, L. A., et al. (2020). Density fluctuations in the solar wind based on type III radio bursts observed by Parker Solar Probe. Astrophys. J. Suppl. 246, 57. doi:10.3847/ 1538-4365/ab65bd

Kundu, M. R., and Haddock, F. T. (1960). A relation between solar radio emission and polar cap absorption of cosmic noise. Nature. 186, 610-613. doi:10.1038/186610a0

Kundu, M. R. (1965). Solar radio Astronomy. New York, NY: Interscience Publishers.

Laitinen, T., Klein, K.-L., Kocharov, L., Torsti, J., Trottet, G., Bothmer, V., et al. (2000). Solar energetic particle event and radio bursts associated with the 1996 July 9 flare and coronal mass ejection. Astron. Astrophys. 360, 729-741.

Laitinen, T., Kopp, A., Effenberger, F., Dalla, S., and Marsh, M. S. (2016). Solar energetic particle access to distant longitudes through turbulent field-line meandering. Astron. Astrophys. 591, A18. doi:10.1051/0004-6361/201527801

Larson, D. E., Lin, R. P., McTiernan, J. M., McFadden, J. P., Ergun, R. E., McCarthy, M., et al. (1997). Tracing the topology of the October 18-20, 1995, magnetic cloud with $\sim 0.1-10^{2} \mathrm{keV}$ electrons. Geophys. Res. Lett. 24, 1911-1914. doi:10. 1029/97GL01878

Leblanc, Y., Dulk, G. A., Kaiser, M. L., and Bougeret, J. (1999). Type U burst and CME in the solar wind. Geophys. Res. Lett. 26, 1089-1092.

Lhotka, C., and Narita, Y. (2019). Kinematic models of the interplanetary magnetic field. Ann. Geophys. 37, 299-314. doi:10.5194/angeo-37-299-2019

Li, B., Cairns, I. H., Gosling, J. T., Malaspina, D. M., Neudegg, D., Steward, G., et al. (2016). Comparisons of mapped magnetic field lines with the source path of the 7 April 1995 type III solar radio burst. J. Geophys. Res. 121, 6141-6156. doi:10. 1002/2016JA022756

Liewer, P. C., Neugebauer, M., and Zurbuchen, T. (2004). Characteristics of activeregion sources of solar wind near solar maximum. Sol. Phys. 223, 209-229. doi:10.1007/s11207-004-1105-z

Lin, R. P. (1985). Energetic solar electrons in the interplanetary medium. Sol. Phys. $100,537-561$.

Lin, R. P., Evans, L. G., and Fainberg, J. (1973). Simultaneous observations of fast solar electrons and type III radio burst emission near 1 AU. Astrophys. Lett. 14, 191.

Maia, D., and Pick, M. (2004). Revisiting the origin of impulsive electron events: coronal magnetic restructuring. Astrophys. J. 609, 1082-1097. doi:10.1086/386319

Malandraki, O. E., and Crosby, N. B. (2018). "Solar energetic particles and space weather: science and applications," in Solar particle radiation storms forecasting and analysis: the HESPERIA HORIZON 2020 project and beyond. Editors O. E. Malandraki and N. B. Crosby (Cham, Switzerland: Springer International Publishing), 1-26. doi:10.1007/978-3-319-60051-2_1

Mann, G., Breitling, F., Vocks, C., Aurass, H., Steinmetz, M., Strassmeier, K. G., et al. (2018). Tracking of an electron beam through the solar corona with LOFAR. Astron. Astrophys. 611, A57. doi:10.1051/0004-6361/201629017

Manning, R., and Fainberg, J. (1980). A new method of measuring radio source parameters of a partially polarized distributed source from spacecraft observations. Space Sci. Instrum. 5, 161-181.

Martínez Oliveros, J. C., Castillo, S. M. D., Krupar, V., Pulupa, M., Bale, S. D., and Calvo-Mozo, B. (2020). An in situ interplanetary "U-burst": observation and results. Astrophys. J. 897, 170. doi:10.3847/1538-4357/ab96c3

Martínez-Oliveros, J. C., Lindsey, C., Bale, S. D., and Krucker, S. (2012). Determination of electromagnetic source direction as an eigenvalue problem. Sol. Phys. 279, 153-171. doi:10.1007/s11207-012-9998-4

Mason, G. M., Mazur, J. E., Dwyer, J. R., Jokipii, J. R., Gold, R. E., and Krimigis, S. M. (2004). Abundances of heavy and ultraheavy ions in ${ }^{3} \mathrm{He}$-rich solar flares. Astrophys. J. 606, 555-564. doi:10.1086/382864

Masson, S., Aulanier, G., Pariat, E., and Klein, K.-L. (2012). Interchange sliprunning reconnection and sweeping SEP beams. Sol. Phys. 276, 199-217. doi:10. 1007/s11207-011-9886-3

Miteva, R., Samwel, S. W., and Costa-Duarte, M. V. (2018). Solar energetic particle catalogs: assumptions, uncertainties and validity of reports. J. Atmos. Sol. Terr. Phys. 180, 26-34. doi:10.1016/j.jastp.2017.05.003

Miteva, R., Samwel, S. W., and Krupar, V. (2017). Solar energetic particles and radio burst emission. J Space Weather Space Clim. 7, A37. doi:10.1051/swsc/2017035

Nindos, A. (2020). Incoherent solar radio emission. Front. Astron. Space Sci. 7, 57. doi: $10.3389 /$ fspas.2020.00057

Nitta, N. V., and DeRosa, M. L. (2008). A comparison of solar open field regions found by type III radio bursts and the potential field source surface model. Astrophys. J. Lett. 673, L207. doi:10.1086/527548
Nitta, N. V., Mason, G. M., Wang, L., Cohen, C. M. S., and Wiedenbeck, M. E. (2015). Solar sources of ${ }^{3} \mathrm{He}$-rich solar energetic particle events in solar cycle 24. Astrophys. J. 806, 235. doi:10.1088/0004-637X/806/2/235

Nitta, N. V., Reames, D. V., DeRosa, M. L., Liu, Y., Yashiro, S., and Gopalswamy, N. (2006). Solar sources of impulsive solar energetic particle events and their magnetic field connection to the Earth. Astrophys. J. 650, 438-450. doi:10.1086/ 507442

Owens, M. J., and Forsyth, R. J. (2013). The heliospheric magnetic field. Living Rev. Sol. Phys. 10, 5. doi:10.12942/lrsp-2013-5

Pacheco, D., Agueda, N., Aran, A., Heber, B., and Lario, D. (2019). Full inversion of solar relativistic electron events measured by the Helios spacecraft. Astron. Astrophys. 624, A3. doi:10.1051/0004-6361/201834520

Paesold, G., Benz, A. O., Klein, K.-L., and Vilmer, N. (2001). Spatial analysis of solar type III events associated with narrow band spikes at metric wavelengths. Astron. Astrophys. 371, 333-342. doi:10.1051/0004-6361:20010358

Papaioannou, A., Sandberg, I., Anastasiadis, A., Kouloumvakos, A., Georgoulis, M. K., Tziotziou, K., et al. (2016). Solar flares, coronal mass ejections and solar energetic particle event characteristics. Journal of Space Weather and Space Climate. 6, A42. doi:10.1051/swsc/2016035

Pick, M., and Ji, S. (1986). Type III burst sources and electron beam injection. Sol. Phys. 107, 159-165. doi:10.1007/BF00155349

Pick, M., Mason, G. M., Wang, Y.-M., Tan, C., and Wang, L. (2006). Solar source regions for ${ }^{3} \mathrm{He}$-rich solar energetic particle events identified using imaging radio, optical, and energetic particle observations. Astrophys. J. 648, 1247-1255. doi:10.1086/505926

Pick, M., and van den Oord, G. H. J. (1990). Observations of beam propagation. Sol. Phys. 130, 83-99.

Pick, M., and Vilmer, N. (2008). Sixty-five years of solar radioastronomy: flares, coronal mass ejections and Sun Earth connection. Astron. Astrophys. Rev. 16, 1-153. doi:10.1007/s00159-008-0013-x

Poquérusse, M., Hoang, S., Bougeret, J.-L., and Moncuquet, M. (1996). "UlyssesARTEMIS radio observation of energetic flare electrons," in Solar wind eight. Editors D. Winterhalter, J. Gosling, S. Habbal, W. Kurth, and M. Neugebauer (Melville, NY: Am. Inst. Phys.), 62-65.

Posner, A. (2007). Up to 1-hour forecasting of radiation hazards from solar energetic ion events with relativistic electrons. Space Weather. 5, S05001. doi:10.1029/2006SW000268

Potter, D. W., Lin, R. P., and Anderson, K. A. (1980). Impulsive 2-10 keV solar electron events not associated with flares. Astrophys. J. Lett. 236, L97-L100. doi:10.1086/183206

Ramesh, R., Mugundhan, V., and Prabhu, K. (2020). New evidence for spatiotemporal fragmentation in the solar flare energy release. Astrophys. J. Lett. 889, L25. doi:10.3847/2041-8213/ab6a9c

Raoult, A., Mangeney, A., and Vlahos, L. (1990). An injection model for type III/V bursts in solar flares. Astron. Astrophys. 233, 229-234.

Reames, D. V. (1999). Particle acceleration at the sun and in the heliosphere. Space Sci. Rev. 90, 413-491.

Reames, D. V. (2015). Solar energetic particles. Cham, Switzerland: Springer.

Reid, H. A. S. (2020). A review of recent solar type III imaging spectroscopy. Front. Astron. Space Sci. 7, 56. doi:10.3389/fspas.2020.00056

Reid, H. A. S., and Kontar, E. P. (2013). Evolution of the solar flare energetic electrons in the inhomogeneous inner heliosphere. Sol. Phys. 285, 217-232. doi:10.1007/s11207-012-0013-x

Reid, H. A. S., and Kontar, E. P. (2017). Imaging spectroscopy of type U and J solar radio bursts with LOFAR. Astron. Astrophys. 606, A141. doi:10.1051/00046361/201730701

Reid, H. A. S., and Kontar, E. P. (2015). Stopping frequency of type III solar radio bursts in expanding magnetic flux tubes. Astron. Astrophys. 577, A124. doi:10. 1051/0004-6361/201425309

Reid, H. A. S., Vilmer, N., and Kontar, E. P. (2014). The low-high-low trend of type III radio burst starting frequencies and solar flare hard X-rays. Astron. Astrophys. 567, A85. doi:10.1051/0004-6361/201321973

Reiner, M. (2001). Kilometric type III radio bursts, electron beams, and interplanetary density structures. Space Sci. Rev. 97, 129-139. doi:10.1023/A: 1011894631803

Reiner, M. J., Fainberg, J., Kaiser, M. L., and Stone, R. G. (1998). Type III radio source located by Ulysses/Wind triangulation. J. Geophys. Res. 103, 1923-1932. doi:10.1029/97JA02646 
Reiner, M. J., Fainberg, J., and Stone, R. G. (1995). Large-scale interplanetary magnetic field configuration revealed by solar radio bursts. Science 270 , 461-464. doi:10.1126/science.270.5235.461

Reiner, M. J., Goetz, K., Fainberg, J., Kaiser, M. L., Maksimovic, M., Cecconi, B., et al. (2009). Multipoint observations of solar type III radio bursts from STEREO and Wind. Sol. Phys. 259, 255-276. doi:10.1007/s11207-009-9404-z

Reiner, M. J., Karlický, M., Jiřička, K., Aurass, H., Mann, G., and Kaiser, M. L. (2000). On the solar origin of complex type III-like radio bursts observed at and below $1 \mathrm{MHz}$. Astrophys. J. 530, 1049-1060.

Reiner, M. J., and MacDowall, R. J. (2015). Electron exciter speeds associated with interplanetary type III solar radio bursts. Sol. Phys. 290, 2975-3004. doi:10. 1007/s11207-015-0779-8

Reiner, M. J., and Stone, R. G. (1986). A new method for reconstructing type-III trajectories. Sol. Phys. 106, 397-401. doi:10.1007/BF00158504

Reiner, M. J., and Stone, R. G. (1988). Model interpretation of type III radio burst characteristics. I - spatial aspects. Astron. Astrophys. 206, 316-335.

Reiner, M. J., and Stone, R. G. (1989). Model interpretation of type III radio burst characteristics. II - temporal aspects. Astron. Astrophys. 217, 251-269.

Richardson, I. G., von Rosenvinge, T. T., Cane, H. V., Christian, E. R., Cohen, C. M. S., Labrador, A. W., et al. (2014). $>25 \mathrm{MeV}$ proton events observed by the high energy telescopes on the STEREO A and B spacecraft and/or at Earth during the first seven years of the STEREO mission. Sol. Phys. 289, 3059-3107. doi:10. 1007/s11207-014-0524-8

Rieger, E., Treumann, R. A., and Karlický, M. (1999). The radio-silent start of an intense solar gamma-ray flare. Sol. Phys. 187, 59-75.

Roelof, E. C., and Pick, M. (1989). Type III radio bursts in a fibrous corona. Astron. Astrophys. 210, 417-424.

Saint-Hilaire, P., Vilmer, N., and Kerdraon, A. (2013). A decade of solar type III radio bursts observed by the Nançay Radioheliograph 1998-2008. Astrophys. J. 762, 60. doi:10.1088/0004-637X/762/1/60

Sakurai, K., and Maeda, H. (1961). A relation between solar radio emission and low-energy solar cosmic rays. J. Geophys. Res. 66, 1966-1969. doi:10.1029/ JZ066i006p01966

Schmahl, E. J., Schmelz, J. T., Saba, J. L. R., Strong, K. T., and Kundu, M. R. (1990). Microwave and X-ray observations of a major confined solar flare. Astrophys. J. 358, 654. doi:10.1086/169018

Schwadron, N. A., Cooper, J. F., Desai, M., Downs, C., Gorby, M., Jordan, A. P., et al. (2017). Particle radiation sources, propagation and interactions in deep space, at Earth, the Moon, Mars, and beyond: examples of radiation interactions and effects. Space Sci. Rev. 212, 1069-1106. doi:10.1007/s11214-017-0381-5

Shih, A. Y., Lin, R. P., and Smith, D. M. (2009). RHESSI observations of the proportional acceleration of relativistic $>0.3 \mathrm{MeV}$ electrons and $>30 \mathrm{MeV}$ protons in solar flares. Astrophys. J. Lett. 698, L152-L157. doi:10.1088/0004637X/698/2/L152

Simnett, G. M. (2017). Energetic particles in the heliosphere. Cham, Switzerland: Springer. Sinclair Reid, H. A., and Ratcliffe, H. (2014). A review of solar type III radio bursts. Res. Astron. Astrophys. 14, 773. doi:10.1088/1674-4527/14/7/003

Smith, E. (2008). "The global heliospheric magnetic field," in The heliosphere through the solar activity cycle. Editors A. Balogh, L. J. Lanzerotti, and S. T. Suess (New York, NY: Springer-Praxis Books), 79-150.

Strauss, R. D., Dresing, N., Kollhoff, A., and Brüdern, M. (2020). On the shape of SEP electron spectra: the role of interplanetary transport. Astrophys. J. 897, 24. doi:10.3847/1538-4357/ab91b0

Tan, B., Mészárosová, H., Karlický, M., Huang, G., and Tan, C. (2016). Microwave type III pair bursts in solar flares. Astrophys. J. 819, 42. doi:10.3847/0004-637X/819/1/42

Thalmann, J. K., Su, Y., Temmer, M., and Veronig, A. M. (2015). The confined $\mathrm{X}$-class flares of solar active region 2192. Astrophys. J. Lett. 801, L23. doi:10. 1088/2041-8205/801/2/L23

Trottet, G., Chupp, E. L., Marschhaeuser, H., Pick, M., Soru-Escaut, I., Rieger, E., et al. (1994). A comparison of gamma-ray and radio emissions during the 11:42 UT solar flare on 1982 June 3. Astron. Astrophys. 288, 647-655.

Trottet, G., Samwel, S., Klein, K.-L., Dudok de Wit, T., and Miteva, R. (2015). Statistical evidence for contributions of flares and coronal mass ejections to major solar energetic particle events. Sol. Phys. 290, 819-839. doi:10.1007/ s11207-014-0628-1

Trottet, G., Vilmer, N., Barat, C., Benz, A., Magun, A., Kuznetsov, A., et al. (1998). A multiwavelength analysis of an electron-dominated gamma-ray event associated with a disk solar flare. Astron. Astrophys. 334, 1099-1111.
Vainio, R., Valtonen, E., Heber, B., Malandraki, O. E., Papaioannou, A., Klein, K.L., et al. (2013). The first SEPServer event catalogue $\sim 68-\mathrm{MeV}$ solar proton events observed at $1 \mathrm{AU}$ in 1996-2010. J. Spa. Wea. Spa. Clim. 3, A12. doi:10. 1051/swsc/2013030

Veronig, A. M., and Brown, J. C. (2004). A coronal thick-target interpretation of two hard X-ray loop events. Astrophys. J. Lett. 603, L117-L120. doi:10.1086/383199

Vestrand, W. T., Share, G. H., Murphy, J. R., Forrest, D. J., Rieger, E., Chupp, E. L., et al. (1999). The Solar Maximum Mission atlas of gamma-ray flares. Astrophys. J. Suppl. 120, 409-467. doi:10.1086/313180

Vilmer, N. (2011). "Contributions of radioheliograph observations to the understanding of solar flares, coronal mass ejections, electron beams in the corona and in the interplanetary medium," in The sun, the solar wind, and the Heliosphere. Editors M. P. Miralles and J. Sánchez Almeida (Cham, Switzerland: Springer), 247

Vilmer, N., Krucker, S., and Lin, R. P.The RHESSI Team (2002). Hard X-ray and metric/decimetric radio observations of the 20 February 2002 solar flare. Sol. Phys. 210, 261-272. doi:10.1023/A:1022492414597

Vilmer, N., MacKinnon, A. L., and Hurford, G. J. (2011). Properties of energetic ions in the solar atmosphere from $\gamma$-ray and neutron observations. Space Sci. Rev. 159, 167-224. doi:10.1007/s11214-010-9728-x

Vlahos, L., Anastasiadis, A., Papaioannou, A., Kouloumvakos, A., and Isliker, H. (2019). Sources of solar energetic particles. Philos. Trans. R. Soc. London, Ser. A. 377, 20180095. doi:10.1098/rsta.2018.0095

Vourlidas, A., Carley, E. P., and Vilmer, N. (2020). Radio observations of coronal mass ejections: space weather aspects. Front. Astron. Space Sci. 7, 43. doi:10. 3389/fspas.2020.00043

Wang, L., Krucker, S., Mason, G. M., Lin, R. P., and Li, G. (2016). The injection of ten electron $/{ }^{3} \mathrm{He}$-rich SEP events. Astron. Astrophys. 585, A119. doi:10.1051/ 0004-6361/201527270

Wang, L., Lin, R. P., Krucker, S., and Gosling, J. T. (2006a). Evidence for double injections in scatter-free solar impulsive electron events. Geophys. Res. Lett. 33, L03106. doi:10.1029/2005GL024434

Wang, Y.-M., Pick, M., and Mason, G. M. (2006b). Coronal holes, jets, and the origin of ${ }^{3}$ He-rich particle events. Astrophys. J. 639, 495-509. doi:10.1086/499355

Wang, L., Lin, R. P., and Krucker, S. (2011). Pitch-angle distributions and temporal variations of $0.3-300 \mathrm{keV}$ solar impulsive electron events. Astrophys. J. 727, 121. doi:10.1088/0004-637X/727/2/121

Wang, L., Lin, R. P., Krucker, S., and Mason, G. M. (2012). A statistical study of solar electron events over one solar cycle. Astrophys. J. 759, 69. doi:10.1088/ 0004-637X/759/1/69

Wang, Y., and Zhang, J. (2007). A comparative study between eruptive X-class flares associated with coronal mass ejections and confined X-class flares. Astrophys. J. 665, 1428-1438. doi:10.1086/519765

Weber, R. R., Fitzenreiter, R. J., Novaco, J. C., and Fainberg, J. (1977). Interplanetary baseline observations of type III solar radio bursts. Sol. Phys. 54, 431-439. doi:10.1007/BF00159934

White, S. M., Benz, A. O., Christe, S., Fárník, F., Kundu, M. R., Mann, G., et al. (2011). The relationship between solar radio and hard X-ray emission. Space Sci. Rev. 159, 225-261. doi:10.1007/s11214-010-9708-1

Wild, J. P. (1968). Radio evidence of a coronal instability before the onset of a solar flare (9 June 1968). Proc. Astron. Soc. Aust. 1, 137-138. doi:10.1017/ S1323358000011061

Wild, J. P., Smerd, S. F., and Weiss, A. A. (1963). Solar bursts. Annu. Rev. Astron. Astrophys. 1, 291-366. doi:10.1146/annurev.aa.01.090163.001451

Wimmer-Schweingruber, R. F., Kern, O., and Hamilton, D. C. (1999). On the solar wind composition during the November 1997 solar particle events: WIND/ MASS observations. Geophys. Res. Lett. 26, 3541-3544. doi:10.1029/ 1999GL010676

Conflict of Interest: The author declares that the research was conducted in the absence of any commercial or financial relationships that could be construed as a potential conflict of interest.

Copyright $\odot 2021$ Klein. This is an open-access article distributed under the terms of the Creative Commons Attribution License (CC BY). The use, distribution or reproduction in other forums is permitted, provided the original author(s) and the copyright owner(s) are credited and that the original publication in this journal is cited, in accordance with accepted academic practice. No use, distribution or reproduction is permitted which does not comply with these terms. 